\title{
APORTACIONES AL CONOCIMIENTO DEL PORTUS ILLICITANUS: RESEÑA DE LOS TRABAJOS DE URGENCIA DE 1976. LA TERRA SIGILLATA
}

\author{
ALFREDO GONZALEZ PRATS \\ Universidad de Alicante
}

A la memoria de mi padre

\begin{abstract}
En este trabajo se ofrece una reseña de las excavaciones de urgencia realizadas en 1976 en el Portus Illicitanus, con una visión de las fases culturales que allí se desarrollaron. Se presenta un catálogo de las cerámicas romanas imperiales halladas durante los trabajos, destacando los ejemplares de Clara Lucente y Clara B, así como los vasos con relieves aplicados, documentos que venían siendo considerados raros o inexistentes en esta parte del País Valenciano.
\end{abstract}

\begin{abstract}
A review of the emergency excavations carried out in 1976 at the Portus Ilicitanus is shown in this paper, as well as a view of the cultural stages which developed in that area. There is a catalogue of Imperial Roman ceramics found during the excavations, where the specimens from Clara Lucente and Clara B, had been considered rare or nonexistent in this part of the Valencian country.
\end{abstract}

\section{INTRODUCCION}

A pesar de que el Portus illicitanus jugó un papel de primer orden en la época romana y fue un centro neurálgico del comercio mediterráneo en la Antigüedad en la fachada oriental y sudoriental de la Península Ibérica, son escasas las noticias que aluden directamente a semejante lugar. Esta parquedad de las fuentes antiguas viene a afectar en menor grado a la colonia romana en función de la cual se constituyó o, mejor, afianzó su puerto comercial, es decir, Illici, poseyendo reiteradas citas en las fuentes de época visigoda al detentar una sede episcopal.

En segundo lugar, así como la Alcudia de Elche ha promovido desde el siglo pasado una creciente labor investigadora por parte de estudiosos locales y extranjeros -recogida por su propietario, el finado Ramos Folqués, y heredada por su hijo, el doctor Ramos Fernández, quien dedicó su tesis, al estudio del yacimiento- a la hora de querer presentar una visión coherente de lo que fue su puerto nos enfrentamos con grandes e insalvables lagunas, que sóló en los últimos tiempos parece que empiezan a rellenarse con el programa de excavaciones sistemáticas emprendidas en la zona por el Museo Arqueológico de Santa Pola, cuya directora ya ha adelantado algunos de los más recientes trabajos (SANCHEZ FERNANDEZ, 1983).

En este estado de cosas, hemos considerado oportuno presentar aquí los datos que poseemos sobre el Portus recogiendo algunos trabajos nuestros realizados tras la cam- 
paña de urgencia llevada a cabo en diciembre de 1976, para la que fuimos comisionados por el consejero provincial de Bellas Artes, el doctor Llobregat Conesa, y que permanecían almacenados en espera de su publicación, habiendo únicamente realizado una nota preliminar sobre las lucernas de aquella campaña (GONZALEZ PRATS, 1977).

\section{LAS FUENTES SOBRE EL PORTUS}

Reunimos en este apartado las fuentes antiguas que de una forma u otra hacen alusión al golfo o puerto ilicitano, incluyendo la del Anónimo de Ravenna y de Artemidoro de Efeso por haber llegado la investigación actual a la identificación del Portus con 'Arwvís /Allon.

La primera mención del calificativo de ilicitano para designar la bahía o seno de Santa Pola se halla en la Historia Natural de C. Plinio el Viejo (23-79 DC) en su libro III, 19:

«luego la (región) de Contestania... En la costa que queda está el río Táder e Ilici, colonia inmune, de la cual recibe el nombre el seno ilicitano»

En el siglo II, al describir el litoral contestano en sus Tablas Geográficas, el matemático, geógrafo y astrónomo de Alejandría Ptolomeo utiliza ya la designación de puerto ilicitano:

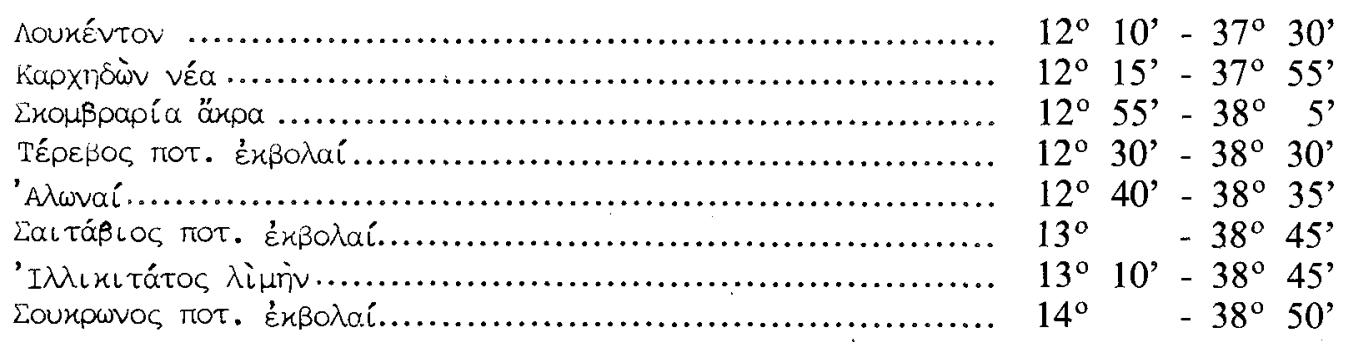

Pomponio Mela, por su parte, en su obra Chorographia describe:

II, 93: «(sinus) sequens Ilicitanus Allonem habet et Lucentiam et unde ei nomen est Ilicem»

Esteban de Bizancio, recoge la cita de Artemidoro de Efeso:

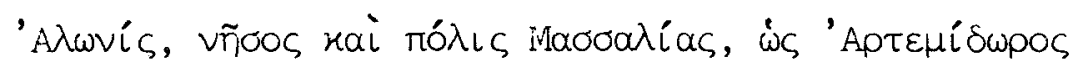

De aquí hemos de pasar al siglo $\mathrm{V}$, en que diversas fuentes recogen la noticia de la destrucción de la flota de Mayoriano en el mes de mayo de 460 por los vándalos. El topónimo citado es Elecem, pero lógicamente se refiere a su puerto. Las citas se hallan en:

HYDATIUS, p. 31, 200:

«Mense Maio Maiorianus Hispanias ingreditur imperator: quo Carthaginiensem provinciam pertendente aliquantas naves, quas sibi ad transitum adversum Vandalos praeparabat, de litore Carthaginiensi commoniti Vandali per proditores abripiunt. Maiorianus ita a sua ordinatione frustratus ad Italiam revertitur.» 
CHRONICA GALLICA, 664 (633-34):

«Maiorianus ingresus Arelatem (qui volens Africam proficisci naves eius in Hispanias a Wandalis captae sunt iuxta Carthaginem Spartariam).»

CHRONICA Marii Epicop. Aventicensis, p. 232:

«His consulibus (Magno y Apolinar) Maiorianus imperator profectus est ad Hispanias. Eo anno captae sunt naves a vandalis ad Elecem iuxta Carthaginem Spartariam.»

ISIDORUS, Historia Vandalorum, 76:

«Maiorianus autem imperator de Italia Spanias veniens cum in Carthaginiensi provincia aliquantas naves sibi ad transitum adversus Wandalos praeparasset, eas de litore Carthaginiensi conmoniti Wandali per proditores abripiunt sicque Maiorianus a sua dispositione frustratus, Italiam revertitur atque a Recimero fraude circumventus occiditur.»

Una referencia bélica de época visigoda la encontramos en la Crónica Mozárabe del 754 , en donde se menciona la victoria del almirante Teodomiro de Oriola rechazando una incursión de la flota bizantina en 700-702. No se alude claramente al Portus, pero dado que el otro gran puerto del litoral, Cartagena, había sido destruido por los godos, el de Santa Pola parece haber servido de base de acción a la flota visigoda:

CONTINUATIO HISP., p. 354, cap. 74:

«...nomine Theudimer qui in Spanie partes non modicas Arabum intulerat neces et, diu exageratos, pacem cum eis federat habiendus. Sed et iam sub Egicane et Witizane Gothorum regibus in Grecis qui equorei nabalique descenderant sua in patria, de palmam victorie triumphaverat.»

Con anterioridad, el Anónimo de Ravenna del siglo VII, que parece basarse en un mapa romano del siglo III, nos ofrece diversos nombres de localidades en esta área geográfica:
IV, 42 pp. 303
5 Saguntum
6 Valentia
7 Portum Sucrone
8 Asterum (Alternum)
9 Setavum (Setabi)
10 Turres
11 Eloe (Edelle)
12 Celeret (Celeri)
13 Dionio
14 Lucentes
15 Leones (Ad Leones)
16 Allon
17 Hilice (Ilice) 
Esta fuente ha dado pie a Llobregat a postular la identificación Allon = Portus Illicitanus (LLOBREGAT, 1983).

Posteriormente, las alusiones al puerto de Santa Pola provienen de geógrafos musulmanes como Al-Himyari, del siglo XIV, quien recoge las citas de AL-UDRI y de ALIDRIDI (s. XI y XII):

AL-HIMYARI: Kitab ar-rawd al-Mi'tar (p. 70 de la versión castellana):

«El distrito de Elche forma parte del círculo de Tudmir, y su cabeza de partido se encuentra a quince millas de Orihuela.

Elche es una gran ciudad situada en una llanura; está atravesada por un brazo del río vecino, que pasa bajo las murallas y abastece las termas, atravesando tiendas y calles; su agua es salobre.

De Elche a Alicante hay veinticinco millas. Se cuenta, entre ot ras cosas, que en el fondeadero llamado de Santa Pola, sobre la orilla cercana a Elche, hay piedras...»

En el siglo XVI poseemos una inscripción en lápida de mármol que estaba situada en el castillo de Santa Pola y en donde se mencionaba no el nombre actual, sino el referente al Puerto de Elche. Según Cristóbal Sanz se leía:

«Bernardino de Cárdenas, duque de Maqueda, lugarteniente del Potentísimo Filipo, Rey de las Españas. Por refugio de los navegantes y de la república christiana edificó este alcázar y castillo del puerto antiguo de Elche. Año 1557» (IBARRA, 1897).

En el siglo XVII, Gaspar Escolano redacta sus Décadas, en cuyo tomo II, libro VI, p. 26, al tratar de Santa Pola dice:

«En este sitio estaba el famoso puerto a quien llamaron los antiguos illicitano, que dio nombre al seno: y aquí mismo tuvo su asiento Illice la Vieja, que se la dio al puerto, como lo atestigua Pomponio Mela, y fue tan nombrada de los antiguos geógrafos cuanto poco atinada de los modernos. Descúbrese hoy día aquel puerto a tiro de arcabuz del castillo de Santa Pola, un grande aljibe donde estaba sita la ciudad. Y cerca del aljibe, al Poniente, muchos rastros del puerto en seco, más de quinientos pasos la tierra adentro, por haberse retirado el mar, como cada día lo experimentamos en la costa.»

Y así, ya hay que pasar a finales del siglo XIX y principios de éste con los datos de tipo arqueológico que nos proporcionan los hermanos Aureliano y Pedro Ibarra, alrededor de La Cenia, que detallaremos en el apartado siguiente.

\section{HISTORIA DE LA INVESTIGACION}

De quienes se han dedicado durante estas dos últimas centurias al estudio y a la recolección de datos sobre el Portus Illicitanus, constituyendo la base de partida para la moderna investigación arqueológica, destaca en primer lugar Aureliano Ibarra, con su obra Illici, su situación y antigüedades, publicada en Alicante en 1879. Allí se recogen diversas noticias, así como hallazgos propios referentes al Portus romano de Santa Pola. Los más importantes se detallan a continuación: 
- En 1602, en el Cap del Aljup y Castillo de Santa Pola se hallaron 52 monedas de Filippo.

- Hallazgo de una pátera o catino de vidrio, decorada en el centro con la cruz de tipo cristiano.

- En las excavaciones realizadas por el marqués de Lendínez se halló:

- una pequeña Victoria de bronce;

- una Venus acéfala de alabastro;

- sortija de oro con gema grabada;

- lucernas;

- monedas;

- ponderales;

- barros saguntinos (t. sigillata);

- dolia y ánforas.

- Hallazgo de un gran sarcófago de mármol con bajorrelieves, así como medallones de mármol con cabezas esculpidas.

- El lugar de La Cenia era conocido desde siempre como «bancal dels chavos» en orden a la ingente cantidad de monedas de bronce halladas.

- Hallazgo de un dolium con la siguiente inscripción de volumen: MXXIII. XXIIX.

Con el título Elche, materiales para su historia, publicado en Cuenca en 1926, Pedro Ibarra continúa la obra y comparte la preocupación hacia el Portus con su hermano Aureliano. Como hiciera éste, recoge datos útiles acompañados de preciosos hallazgos en la zona objeto de posteriores excavaciones, como:

- Los hallazgos de 1898 en La Cenia, traducidos en:

- un collar de cuentas y broche de oro;

- áureo de MAYORIANO (457-461): D.N. NLIVS MAIORIANVS PPAVE;

- sortija que en su chatón llevaba una moneda de MARCO AURELIO (161-180): ANTONINVS AVG. ARMENIACVS;

- collar de oro con cuentas de ágata;

- dos áureos de GALIENO (253-265): GALLIENVS P.F. AVS, GALLIENVS AVG;

- un áureo de ARCADIO (395-408): D.N. ARCADIVS PF AVG.

- En 1929, adquiere un áureo de HONORIO.

- En 1885, junto al cementerio, se halló gran cantidad de fragmentos de bronce, al parecer de una estatua de Augusto.

- Noticia, recogida a su vez de un manuscrito anónimo de 1752, de una lápida de cuatro palmos y cuatro dedos por dos palmos y dos dedos en uno de los muros del castillo de Santa Pola con la siguiente inscripción:

D.M.

VLP. MARCIANAE

VIXIT AN. XXX

L. CASSIVS. IVNIA

NVS. MARITAE

KARISSIMAE. 
- Los hallazgos conjuntos con el señor Múrtula en 1914 en La Cenia:

- molde de tres sellos abiertos en placa de pizarra;

- disco de plomo con un medallón con una cabeza varonil en relieve barbada y con diadema;

- fragmento de escultura (hombro derecho y pecho de un niño);

- paloma de cerámica de pasta clara;

- fragmentos de mosaicos polícromos;

- una lucerna;

- un vaso de sigillata de la forma DRAG. 27 con la marca OF. RVFI dentro de círculo;

- fragmentos de cerámica ibérica pintada;

- cerámica estampada gris y roja;

- medallón de lucerna con un Cupido sentado jugueteando con un perro.

Posteriormente, A. Ramos Folqués, en su Historia de Santa Pola, viene a recoger todas las anteriores citas y noticias de los hermanos Ibarra en su capítulo VII, que trata del Portus Illicitanus. Las noticias sobre actividades y hallazgos más recientes se resumen en:

- Las excavaciones en 1935 del padre Belda en los alrededores del cementerio, hallando cuatro sepulturas que consideró visigodas.

- Hallazgo de una boca de ánfora con la marca CRAMAR por don Claudio Miralles de Imperial y Gómez en su propiedad.

- Hallazgo por don Francisco Campello García, en los bancales al oeste del cementerio de una sepultura «con cuatro losas de ladrillos» que proporcionó un fragmento de frasco de vidrio, una jarrita de barro amarillento y un fino vaso decorado con relieves alusivos a los trabajos de Hércules.

En sus láminas pueden observarse diversos materiales, entre ellos, cerámicas ibéricas pintadas, cerámicas áticas de figuras rojas, cerámicas claras estampadas y ánforas del tipo Dressel 4 y 7.

\section{LAS EXCAVACIONES DE 1976}

Los trabajos tuvieron carácter de urgencia y salvamento, desarrollándose durante todo el mes de diciembre de 1976 y parte de enero siguiente.

El lugar en que se desarrollaron fue una zona de La Cenia, concretamente la finca a poniente del Palmeral, al otro lado de la calle. En una primera fase, adelantándonos al paso de la destructora pala excavadora, pudimos aflorar y delimitar los restos de tres grandes departamentos o almacenes que arrojaban medidas de $16 \mathrm{~m}$. por $5,5 \mathrm{~m}$. de ancho, con muros de $0,5 \mathrm{~m}$. de espesor. áreas:

Una vez se pudo detener las obras, el campo de trabajo quedó establecido en tres

- La BALSA 1, midiendo su interior 5 por $2,5 \mathrm{~m}$. y su exterior 6 por 3,5 $\mathrm{m}$., con paredes de hormigón de $0,5 \mathrm{~m}$.

- La BALSA 2, que alcanzaba una longitud de 15 por $1,80 \mathrm{~m}$., sin acabarse la misma.

- La ZONA A-B, más septentrional. 

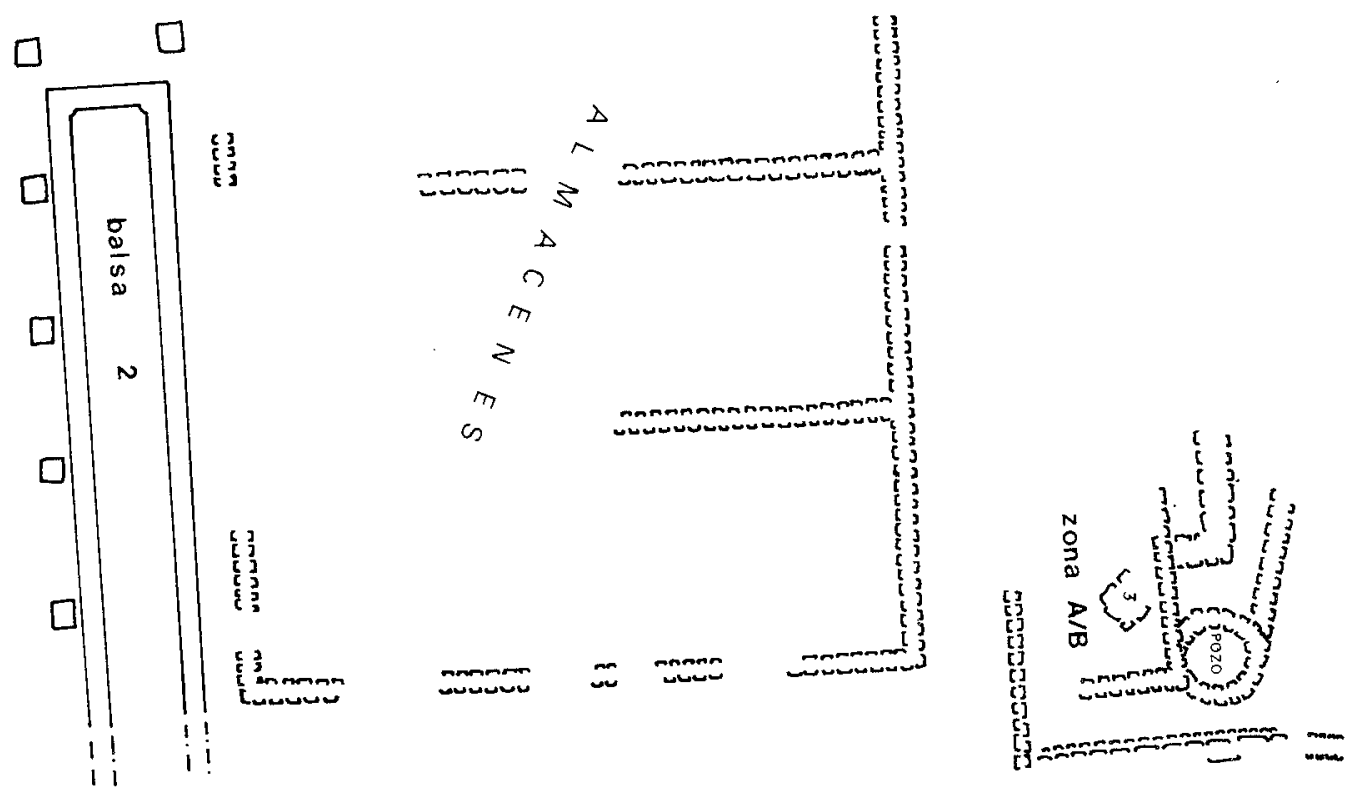

c a rretera

Plano de la excavación de 1976 
Inicialmente, se procedió al vaciado de las dos BALSAS, proporcionando ingente cantidad de materiales: fragmentos de mosaicos policromos, grandes platos o fuentes de mármol, lucernas de diversos tipos, abundantísima cerámica común y ánforas, cerámicas finas de importación (campanienses, itálicas, sudgálicas, hispánicas y un gran volumen de claras, aparte de numerosas monedas de bronce cuyo pésimo estado de conservación no permite una clara identificación.

Las BALSAS se construyeron con el duro OPUS CAEMENTICIUM y presentaban la superficie interna y el reborde superior alisados, con listones redondeados en los ángulos. Su altura conservada viene a alcanzar los $2,5 \mathrm{~m}$.

La BALSA 2 parece continuar al otro lado de la carretera, ya que al efectuar ésta nos notificaron que rompieron un fuerte foso que la cruzaba.

Los materiales, por tanto, se hallan revueltos y oscilan con una cronología del siglo I a. C. al V d. C. Da la impresión de que en un momento tardío del Bajo Imperio y seguramente a partir del siglo VI se rellenaron ambas balsas con escombros que procedían de otro lugar del Portus. En función de qué se realizó este relleno lo ignoramos por el momento. Lo que resulta obvio es que las BALSAS carecían ya de utilidad.

El área en donde se pudo trabajar con más calma pasó a denominarse Zona A y B, separadas por un testigo amplio. Allí se pudo establecer la estratigrafía siguiente:

0. Nivel superficial actual, creado a raíz del terraplenado de los antiguos bancales («dels chavos»), cuya altura venía a coincidir con los dos metros más que presenta el Palmeral.

I. Nivel de tierras grises y negruzcas, con fragmentos de cerámicas claras y lucernas con pico de corazón y agujero de alimentación umbilicado, que se puede fechar en el siglo III d. C. MURO I.

II. Nivel de color marrón anaranjado que ofreció abundante cerámica aretina y sudgálica en menor grado junto con lucernas de volutas de pico triangular, fechable en la primera mitad del s. I d. C. A nivel estructural, aparece el complejo de los MUROS II.

III. Nivel de color más grisáceo subyacente, que proporcionó alguna cerámica ibérica, fragmentos de campaniense B y lucernas preaugusteas del tipo con cabezas de pájaros, lo que nos permite fecharlo hacia la mitad del s. I a. C. Aparecieron restos de muros (MUROS III).

Contigua a esta zona se sondearon tres cortes denominados 1,2 y 3 , en los que se pudo establecer dos niveles:

II. El inferior, correspondiente a parte del vestíbulo de una casa con pavimento de OPUS SIGNINUM, amén de lo que parece ser la cocina. Proporcionó cerámicas sudgálicas y aretinas. Se corresponde con el nivel II anterior.

I. El superior, que clausura la estructura anterior, asentando un muro sobre el pavimento con placas de mármol, y que ofrecía entre otros materiales sigillatas claras, correspondiéndose con el nivel I de la Zona A-B.

Al SE. de ambas BALSAS y, al parecer, encerrando el conjunto, la pala excavadora fue extrayendo regularmente grandes sillares perfectamente escuadrados de forma rectangular o cúbica, que formarán una línea recta y de los que se conservaron los cinco que estaban casi adosados a la BALSA 2.

Igualmente, aprovechando un segundo desmonte de la pala excavadora en el ángulo oriental de la parcela de construcción, se pudo comprobar la existencia de un potente 


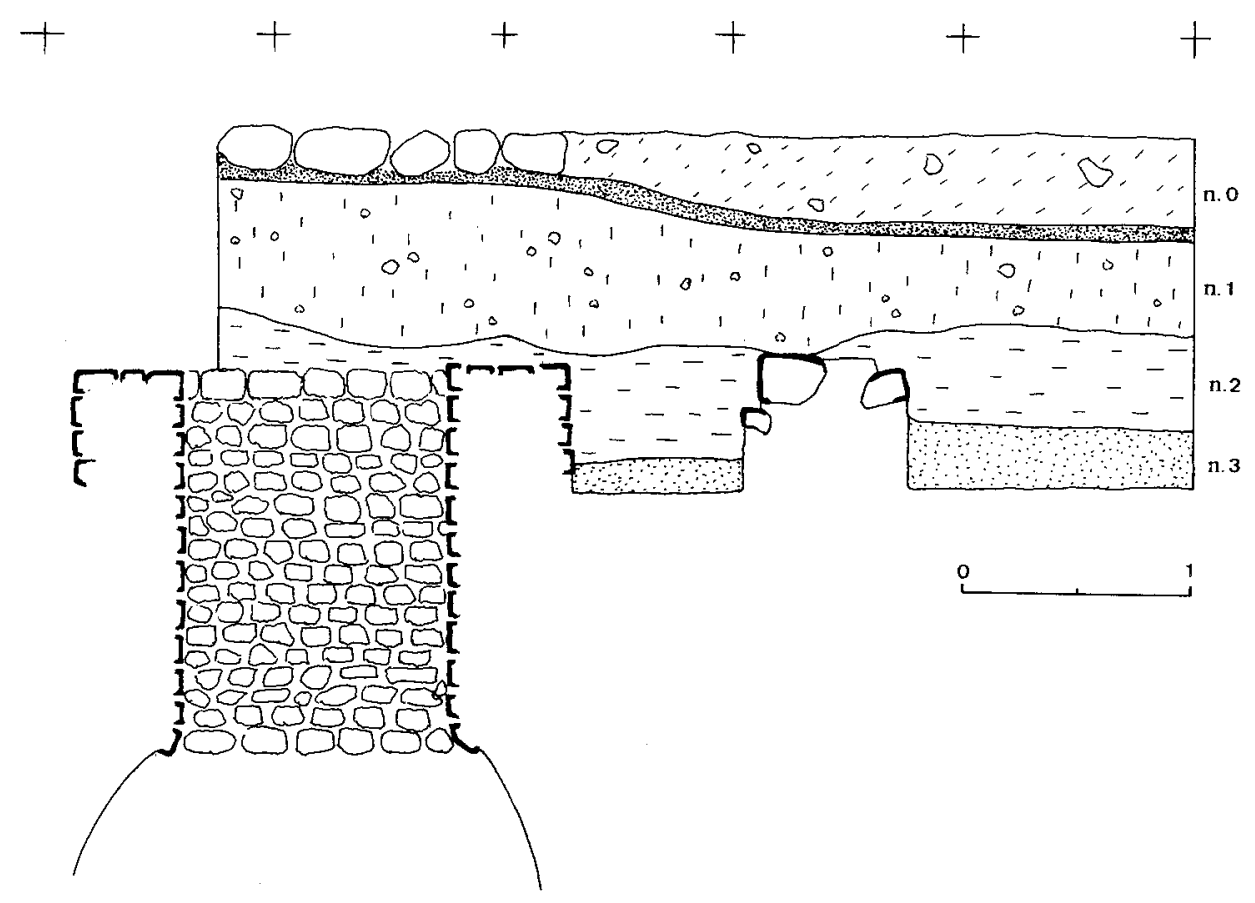

Fig. 2. Estratigrafías de la Zona A.

nivel preaugusteo que ofreció cerámica ibérica pintada del estilo ELCHE-ARCHENA y una forma LAMB. 7 de Campaniense B.

Lo mismo ocurrió en el Aljibe 1, cuya antigua construcción, con paredes de hormigón semejantes a las de las BALSAS, se efectuó cortando un nivel preexistente del que se recogieron cerámicas ibéricas pintadas.

Para confirmar estos hallazgos, con posterioridad a las excavaciones de diciembreenero se realizó un sondeo al oeste del cementerio, evidenciándose con gran sorpresa de todos un rico nivel ibérico contando con buena proporción de cerámica ática de barniz negro, fechable en el s. IV a. C y que por el momento viene a representar la fase más antigua del puerto que a raíz de la fundación de Illici tomó su nombre.

De los materiales exhumados en la campaña de urgencia de La Cenia estamos llevando a cabo - debido a la ingente masa de materiales - varios estudios parciales. Así, en lo que concierne a las lucernas, los tipos aparecidos son los siguientes:

I) Preaugusteos:

a) Con el pico decorado con cabezas de pájaros (DRESSEL-LAMBOGLIA, 4).

b) Delfiniforme, con decoración de glóbulos (DRESSEL-LAMBOGLIA, 2).

II) Imperiales:

a) De volutas sencillas y pico triangular (DRESSEL-LAMBOGLIA, 9 B).

b) De alerones laterales (DENEAUVE, $V$ G).

c) De disco y pico redondo (DRESSEL-LAMBOGLIA, 20).

d) Con pico de corazón y orla decorada (DRESSEL-LAMBOGLIA, 28 B y 30 A-B). 
e) De canal abierto corto y curvo (PONSICH, $4 \mathrm{~A}$ ).

f) De canal largo y recto (PONSICH, IV B).

Con lo que se convierte en un perfecto muestreo de la amplitud de vida del Portus romano, abarcando desde la mitad del s. I a. C. hasta el s. V d. C.

Si tomamos en consideración la distribución porcentual de las cerámicas sigillatas, se desprende la existencia de dos momentos en que el Portus Illicitanus se muestra más activo. El primero hacia la primera mitad del s. I a. C. y hasta la mitad del I d. C., con gran volumen de importaciones de lucernas de volutas y pico triangular, con sigillatas itálicas lisas y decoradas, así como sudgálica en menor grado.

Y otro momento, tal vez desde el último tercio del s. II d. C. hasta el s. V d. D. (sigillatas claras D y estampada, lucernas de canal corto o largo de tipo paleocristiano).

El primer movimiento se puede poner en relación con la creciente y nueva demanda que la recién fundada C. I. I. A. en el año 42 a. C. genera, así como secundariamente por los propios habitantes de la ciudad portuaria.

Los hallazgos muestran que los productos seguían llegando a lo largo de los siglos I, II y III, con un probable estancamiento durante la crisis de mediados de este último siglo, para cobrar nuevamente su actividad a lo largo de los dos siglos siguientes.

La cerámica estampada gris que presenta P. Ibarra - reflejo de las bellas fuentes de Illici- y ciertas curiosas formas de cerámica común parecen marcar la última etapa alrededor de los siglos V y VI d. C., aunque sabemos que el Portus sigue viviendo con carácter comercial y militar hasta el siglo VIII como mínimo, y tal vez el estudio de las cerámicas comunes halladas en las BALSAS pueda documentar tipos que vendrían a cubrir ese vacío.

\section{CONCLUSIONES}

Con los datos proporcionados por Aureliano y Pedro Ibarra, y posteriormente por Ramos Folqués, y con los nuevos extraídos del estudio preliminar de los hallazgos de la campaña de salvamento de diciembre de 1976, podemos resumir el estado de la cuestión acerca de las fases de ocupación del Portus en los siguientes puntos:

1. Existencia de un habitat ibérico antiguo que por sus importaciones griegas puede ser fechado a lo largo de los siglos V-IV a. C. El área que ocupa parece extenderse al oeste del cementerio de Santa Pola.

2. Constatación de un nivel ibérico-republicano avanzado, fechable en la primera mitad del siglo I a. C., debajo de los niveles altoimperiales de La Cenia.

3. Un gran momento de esplendor comercial y urbanístico que se sitúa a partir de la segunda mitad del s. I a. C. El Portus Illicitanus debió decrecer y extenderse generando una verdadera ciudad portuaria que no sólo estaba en función de la demanda de Illici, sino que constituía de por sí un fuerte núcleo de consumo. Este momento continuó ininterrumpidamente con menor volumen de importaciones, al parecer, hasta la crisis del s. III d. C.

4. Paralela a la reconstrucción de que es objeto Illici, tras las destrucciones que acompañan la crisis, renace igualmente la actividad de su puerto. El gran volumen de sigillata clara D y estampada marcan bastante bien esta postrera etapa de vida del Portus, cuyo final no estamos en condiciones de determinar. 

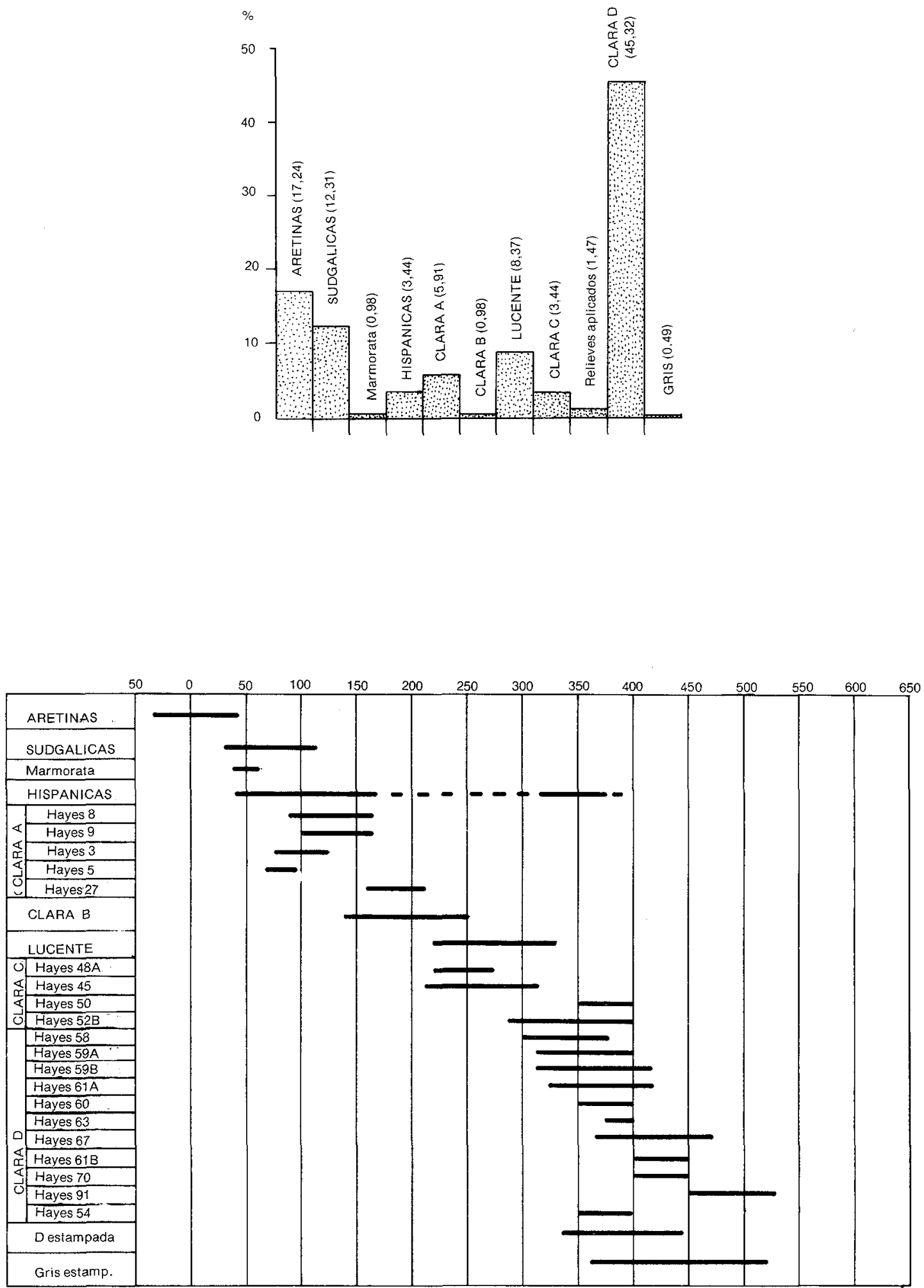

Fig. 3. Histograma de frecuencias de los distintos tipos de terra sigillata y cuadro cronológico. 
Arqueológicamente y con las reservas que debe implicar el extraer datos de una sola excavación, estos cuatro momentos parecen quedar bastante bien definidos. Con todo, la mayor importancia de los trabajos realizados estriba en la posibilidad de determinar la existencia de un asentamiento indígena por los albores del s. V-IV a. C. que creemos ya en función del mejor fondeadero y puerto del litoral contestano.

\section{LA TERRA SIGILLATA}

Este apartado que versa sobre las cerámicas finas de época imperial romana, en realidad incluye dos trabajos distintos realizados tras la campaña de 1976 con el fin de dar a conocer con la mayor rapidez los principales materiales arqueológicos recuperados. Es por ello que el conjunto de sigillatas rojas presenta una homogeneidad en su presentación que difiere del tratamiento que hicimos de la sigillata anaranjada o clara.

Por otro lado, es nuestro propósito presentar únicamente un catálogo de toda la terra sigillata hallada en los trabajos referidos, relegando para el futuro un estudio crítico que pensamos debe realizarse manejando también los últimos documentos aparecidos en las excavaciones que se llevan a cabo actualmente en la zona del Portus. Y, a mayor abundamiento, será imprescindible conocer todo el repertorio de formas y marcas hallados en el resto de la provincia, especialmente en La Alcudia, para llevar a feliz término un trabajo de este tipo y confeccionar las oportunas cartas de distribución y dispersión. En este ánimo, nuestro trabajo no es otro que el de facilitar al especialista el conocimiento de los materiales que se reseñan a continuación.

Dentro del primer grupo de terra sigillata, representado por los productos aretinositálicos, sudgálicos e hispánicos, destaca el mayor porcentaje correspondiente a las cerámicas itálicas, en su mayoría aretinas, que además nos ha permitido conocer las únicas marcas de este trabajo, pues las de las fábricas sudgálicas se presentan ilegibles en una primera apreciación. Estos porcentajes quedan reflejados a continuación:

$\begin{array}{ll}\text { Itálicas: } 51,4 \% & \text { (decoradas: 8,8 \%) } \\ & \text { (lisas: 42,6 \%) } \\ \text { Sudgálicas: } 42,5 \% & \text { (decoradas: 17,6 \%) } \\ & \text { (lisas: 22 \%) } \\ & \text { (marmorata: 2,9\%) }\end{array}$

Hispánicas: $7,3 \%$.

Los talleres localizados a través de las marcas de alfarero son los siguientes, todos aretinos:
CN. ATEIUS
CN. ATEI MAHES
CN. ATEI PLOCAMI
M. GRATIDIUS
RASINIUS
UMBRICIUS
XANTHUS
GELLIUS (?). 

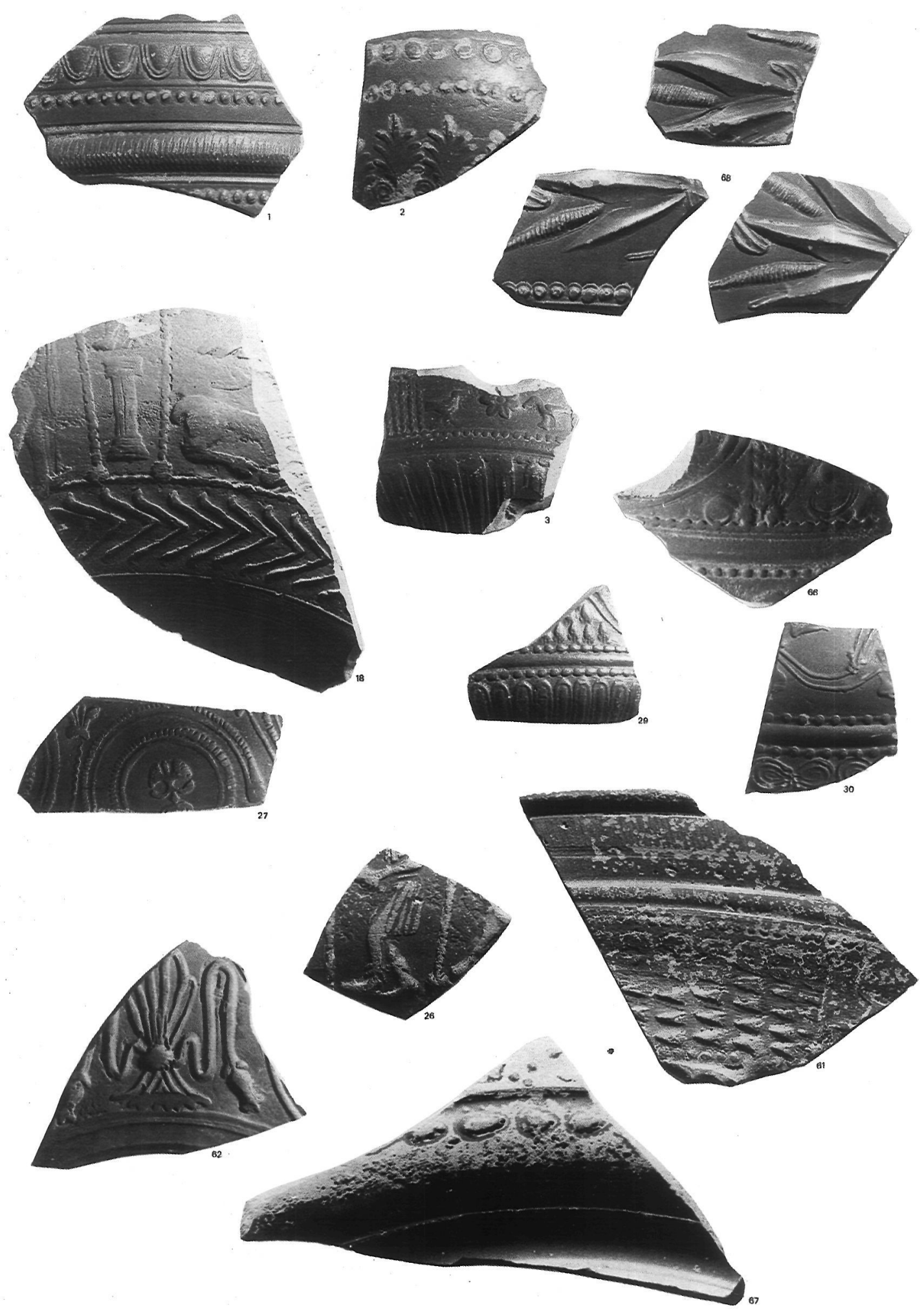

Lám. I. Selección de sigillatas rojas decoradas. 

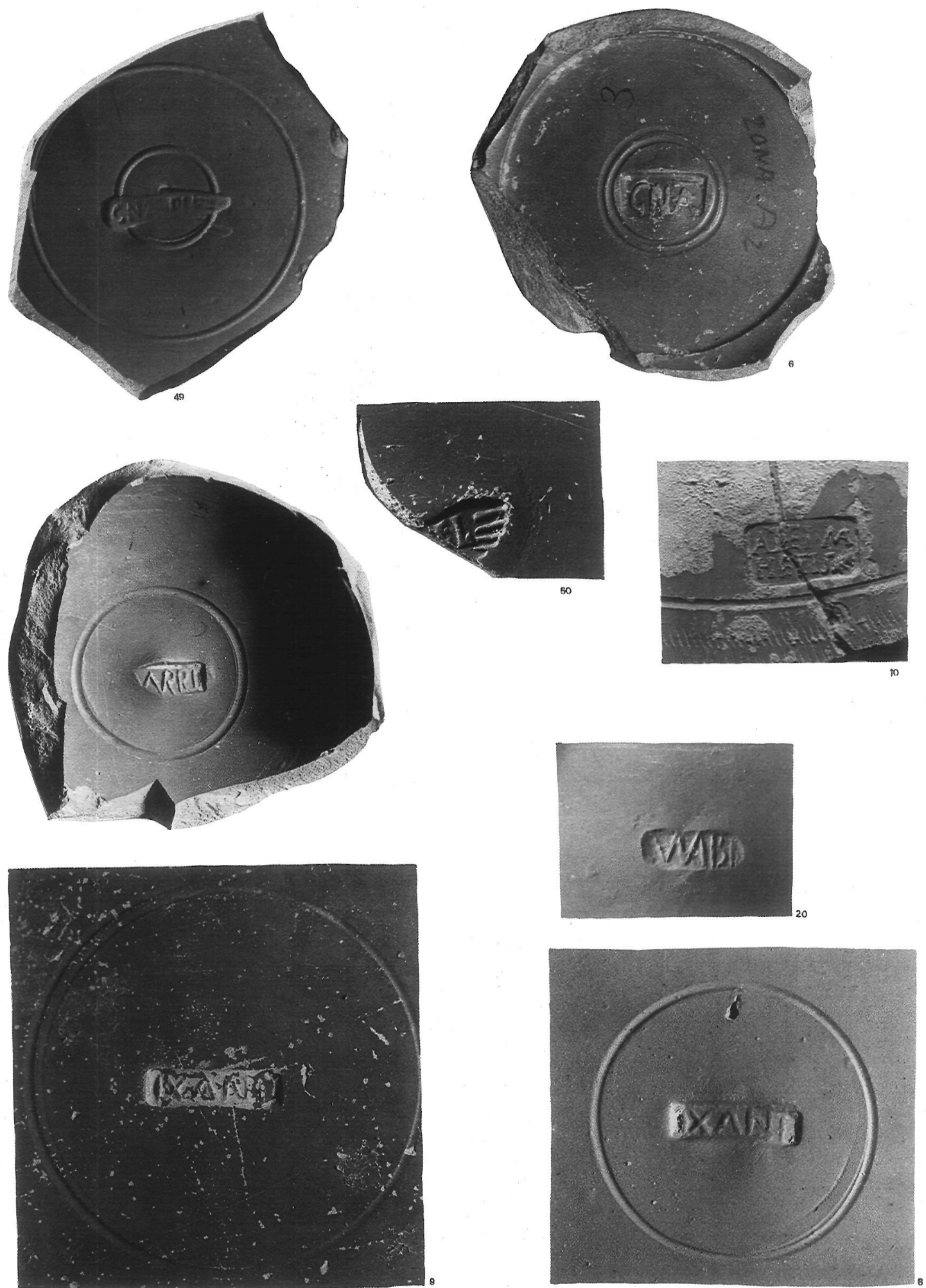

Lám. II. Selección de marcas de alfarero. 


\section{CATALOGO}

\section{Superficie}

1.-Fragmento de pared de una copa aretina decorada de la forma 11. Inv.: SPI-55.

2. - Fragmento de pared inferior de una copa aretina decorada de la forma 11 ó 13 . Inv. SPI-59.

3.-Fragmento de la parte inferior con carena de un vaso DRAG. 29 a de sudgálica decorada. Inv.: SPI-54.

4.-Fragmento de base de un plato de forma DRAG. 31 ó 33 de cerámica aretina lisa. Diámetro de la base: $4,7 \mathrm{~cm}$.

Marca en cartela cuadrangular dentro de circunferencia: M/CRATI. Del taller de M. GRATIDIUS (Oxé, 762).

Inv.: SPI-45.

\section{Zona A}

5.-Base con parte de las paredes de un vaso aretino liso de la forma RITT. 5. Diámetro de la base: $6,7 \mathrm{~cm}$.

Marca en cartela cuadrangular con apéndices circulares en los ángulos: RASI. Del taller de RASINIUS (Oxé, 1485, 25.29). Grafito en el exterior de la base: BOC. Hallado en el nivel II.

Sin inventariar.

6.-Base de una forma HALTERN 7 de cerámica aretina lisa.

Diámetro: $4,2 \mathrm{cms}$.

Marca en cartela rectangular dentro de doble circunferencia: CN. ATE. Del taller de CN. ATEIUS (Oxé, 145). En el exterior del fondo, grafito de trazos tal vez numéricos.

Hallado en el nivel II.

Sin inventariar.

7.-Vaso completo de la forma DRAG. 27 de cerámica aretina lisa.

Diámetro del borde: $11,8 \mathrm{cms}$.

Diámetro de la base: $5,6 \mathrm{cms}$.

Altura: $6,7 \mathrm{cms}$.

Marca en interior de cartela rectangular con los ángulos ligeramente redondeados:

XANTHI. Del taller de XANTHUS (Oxé, 177). Grafito en el fondo externo.

Hallado en el nivel II.

Sin inventariar.

8.-Plato completo de la forma DRAG. 17 a de cerámica aretina lisa.

Diámetro del borde: $16 \mathrm{~cm}$.

Diámetro de la base: $8,7 \mathrm{~cm}$.

Altura: $3,6 \mathrm{~cm}$.

Marca en el centro del fondo interno y dentro de cartela rectangular: IXANTI, con el nexo NT. Corresponde al taller de XANTHUS, que trabajó en el de CN. ATEIUS (Oxé, 176). La I que precede al cognomen podría ser la letra final del cognomen ATEI tal como aparece en las marcas de doble línea (CN. ATE/IXANTHI) (Oxé, 176, 22), habiéndose tomado solamente la última de ellas. Parece ser la única posibilidad, dado que la marca aislada de XANTHUS no aparece nunca precedida de I. 

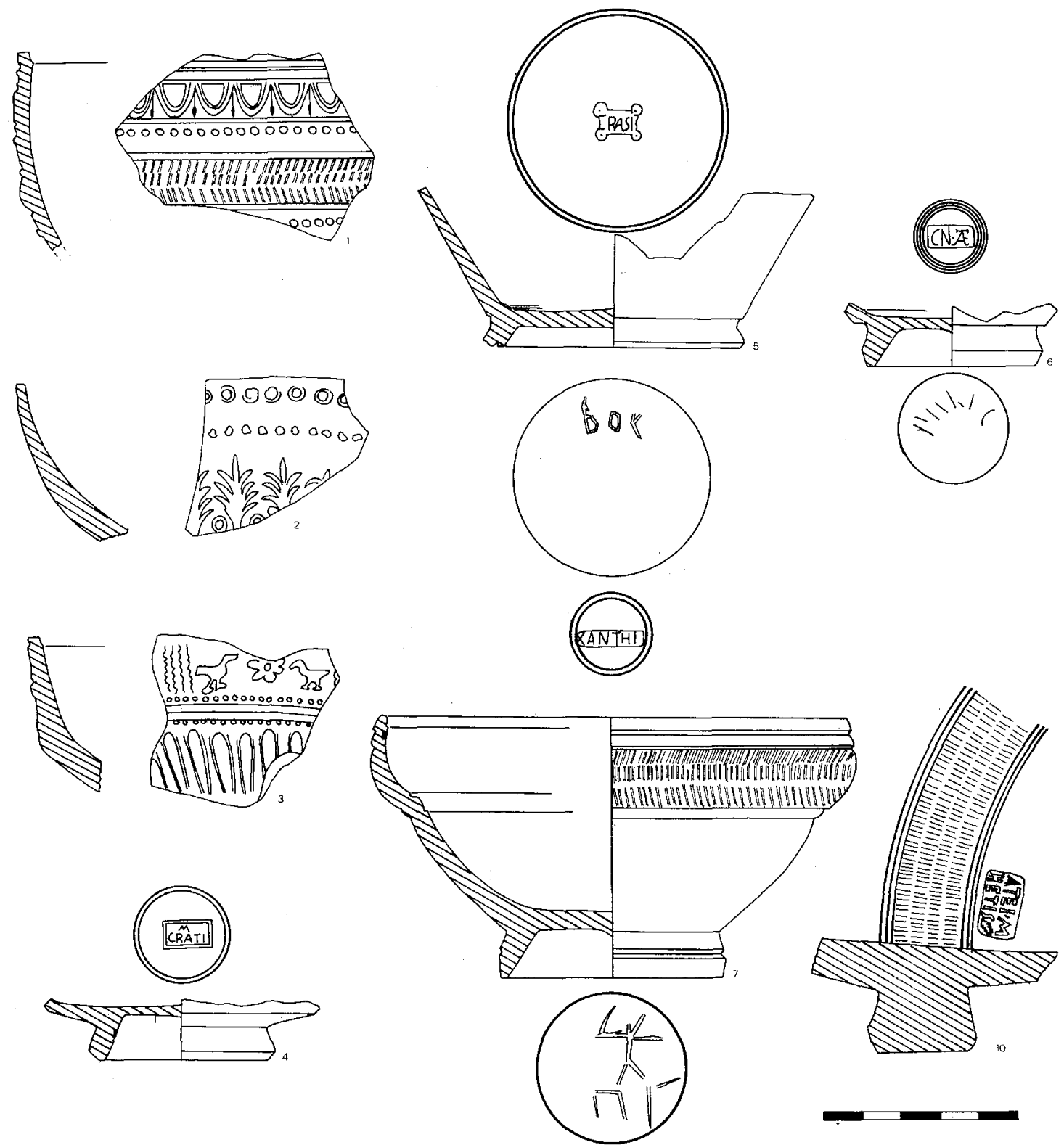

Fig. 4. Terra sigillata roja (aretina). Superficie y nivel II de la Zona A.

En el fondo externo, tres grafitos, uno posiblemente legible.

Hallado en el nivel II.

Sin inventariar.

9.--Plato completo de la forma DRAG. 17 a de cerámica aretina lisa.

Diámetro del borde: $16 \mathrm{~cm}$.

Diámetro de la base: $8,7 \mathrm{~cm}$.

Altura: $3,6 \mathrm{~cm}$.

Marca en el centro del fondo en cartela rectangular: IXANTHI, con el nexo NT. Del taller de XANTHUS. Ejemplar idéntico al anterior, con las mismas consideraciones en torno a la marca.

Hallado en el nivel II.

Sin inventariar. 

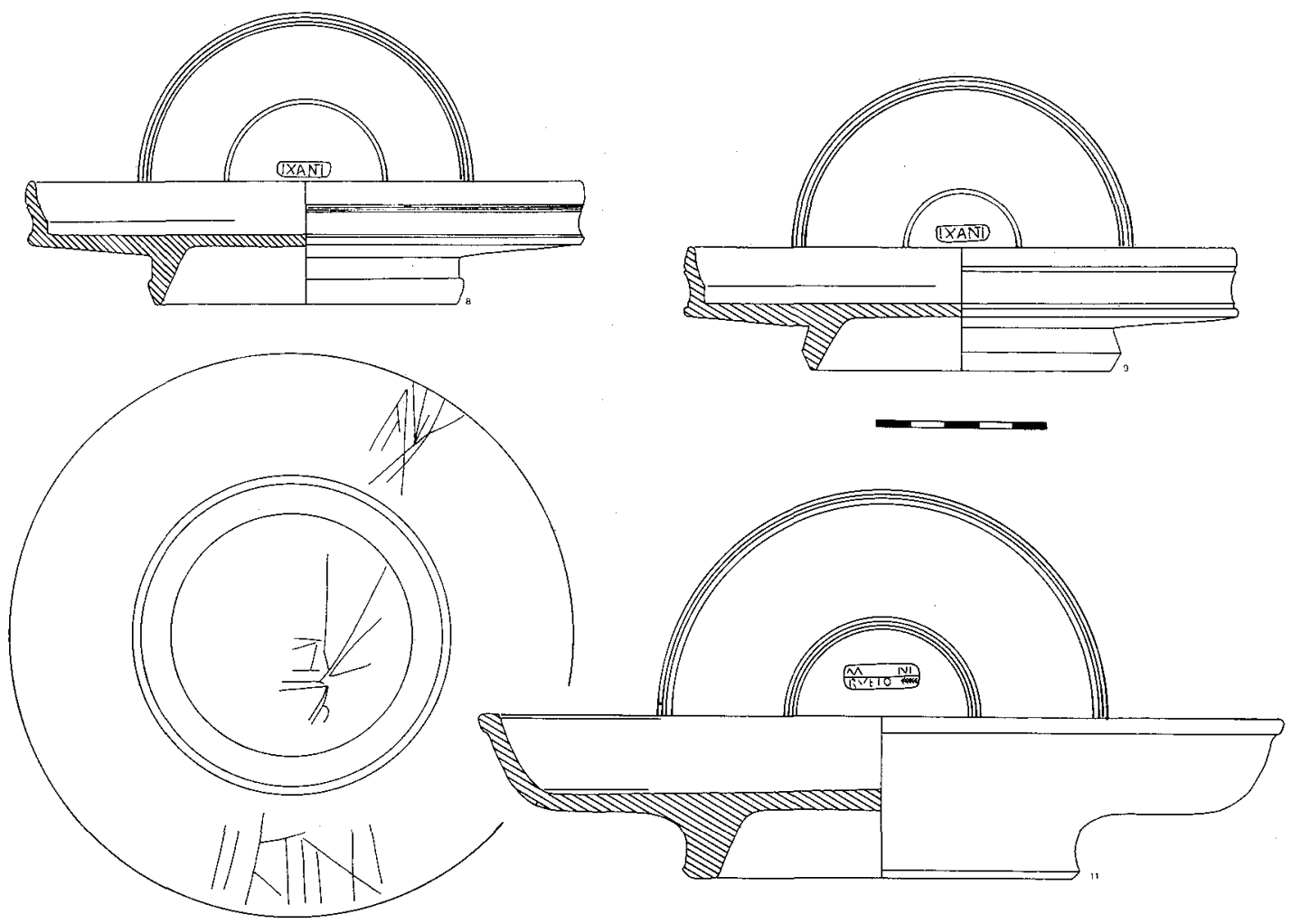

Fig. 5. Terra sigillata roja (aretina). Nivel II de la Zona A.

10.-Tres fragmentos que unen entre sí de una fuente de forma HALTERN I B de cerámica aretina lisa. Orla a ruedecilla y una marca en cartela cuadrangular: ATEIMA/HETIS. Del taller de CN. ATEI MAHES (Oxé, 168; similar a la 8.12, pero con el nexo MA y al final una estrella en lugar de la espiga de este ejemplar).

Procede del nivel II.

Sin inventariar.

11.- Plato de cerámica aretina lisa, de forma parecida a la DRAG. 18.

Diámetro del borde: $23,4 \mathrm{~cm}$.

Diámetro de la base: $11 \mathrm{~cm}$.

Altura: $4,7 \mathrm{~cm}$.

Fondo con dos grupos de dobles circunferencias y en el centro cartela rectangular con ángulos redondeados y marca en doble línea. Ha saltado el barniz y al ser débil la impresión prefiero no aventurar lectura alguna.

Procede del nivel II.

Sin inventariar.

\section{Zona B}

12.-Fragmento de borde y pared de una copa de la forma RITT. 5 de cerámica aretina lisa.

Procede del nivel II.

Sin inventariar. 
13.-Fragmento de borde y pared de un plato de forma HALTERN I A de cerámica aretina lisa.

Procede del nivel II.

Sin inventariar.

14.-Fragmento de borde y pared de una copa HALTERN 7 de cerámica aretina lisa. Procede del nivel II.

Sin inventariar.

15.-Fragmento de borde y pared de una forma DRAG. 27 de cerámica aretina lisa. Procede del nivel II.

Sin inventariar.

16. - Fragmento de fina pared de una copa aretina decorada, tal vez una forma 11 ó 13. Procede del nivel II.

Sin inventariar.

17.-Fragmento de borde de una forma HERMET 19 de cerámica sudgálica lisa. Sin inventariar.

18.-Fragmento de pared de una forma DRAG. 37 de cerámica sudgálida decorada: piernas de figura humana, estatua sobre columna y cuadrúpedo. Sin inventariar.

19.-Base de una copa de cerámica sudgálica lisa, con marca ilegible en cartela rectangular de ángulos redondeados y rebasando la circunferencia en que se encuentra inscrita.

Diámetro de la base: $3,1 \mathrm{~cm}$.

Sin inventariar.

\section{Balsa 1}

20.-Base con parte de la pared de una forma RITT. 5 de cerámica aretina lisa. Diámetro de la base: $6,1 \mathrm{~cm}$.

Marca en cartela oblonga: VMBI, con el nexo VM. Del taller de UMBRICIUS (Oxé, 2385). Grafito en el fondo externo. Inv.: SPI-64.

21.- Fragmento de borde y pared de una copa de la forma RITT. 5 de cerámica aretina lisa. Inv.: SPI-391.

22.-Fragmento de borde y pared de la forma RITT. 9 de cerámica aretina lisa. Inv.: SPI-392.

23.-Fragmento de borde con ruedecilla, de cerámica aretina lisa. Inv.: SPI-400.

24.-Fragmento de borde de una forma RITT. 9 de cerámica aretina lisa. Inv.: SPI-393.

25.-Fragmento de borde de una fuente HALTERN I A de cerámica aretina lisa. Inv.: SPI-394.

26.-Fragmento de cerámica sudgálica o hispánica con personaje humano. Inv.: SPI-154.

27.-Fragmento de pared de una forma DRAG. 29 ó 36 de cerámica hispánica decorada.

Inv.: SPI-177.

28.-Parte de una copa de forma DRAG. $24 / 25$ de cerámica sudgálica lisa. Inv.: SPI-146.

29. - Fragmento de pared con inicio de la carena de una forma DRAG. 29 de cerámica sudgálica decorada.

Inv.: SPI-168. 
30.-Fragmento de pared de una forma DRAG. 29 a de cerámica sudgálica decorada. Inv.: SPI-170.

31.-Fragmento de borde de plato de cerámica sudgálica marmorata. Inv.: SPI-189.

32.-Fragmento de plato de la forma DRAG. 18 (?) de cerámica sudgálica lisa. Inv.: SPI-390.

33. - Fragmento de pared superior de una forma DRAG. 29 de cerámica sudgálica decorada. Inv.: SPI-397.

34.-Fragmento de borde de una forma DRAG. 18 (?) de cerámica sudgálica lisa. Inv.: SPI-395.

35.-Fragmento de borde de una forma DRAG. 18 (?) de cerámica sudgálica lisa. Inv.: SPI-398.

36.-Fragmento de borde de una forma DRAG. 18 (?) de cerámica sudgálica lisa. Inv.: SPI-399.

37. - Fragmento de borde y pared de una forma DRAG. $24 / 25$ de cerámica hispánica. Inv.: SPI-389.

\section{Balsa 2}

38. - Fragmento de borde de un plato DRAG. 17 a de cerámica aretina lisa. Inv.: SPI-429.

39. - Fragmento de borde de una fuente de forma HALTERN I A, de cerámica aretina lisa.

Inv.: SPI-441.

40.-Fragmento de borde de una forma HALTERN I A de cerámica aretina lisa. Inv.: SPI-452.

41.-Fragmento de borde de un plato de la forma DRAG. 17 a de cerámica aretina lisa. Inv.: SPI-433.

42.-Fragmento de pared de una copa aretina decorada de la forma 11. Inv.: SPI-444.

43.-Fragmento de pared de una forma RITT. 5 de cerámica aretina lisa. Inv.: SPI-445.

44.-Fragmento de plato de forma DRAG. 17 a de cerámica aretina lisa. Inv.: SPI-427.

45.-Fragmento de borde de una fuente de forma HALTERN I, de cerámica aretina lisa. Inv.: SPI-459.

46.-Fragmento de borde de una fuente de forma HALTERN I, de cerámica aretina lisa.

47.--Fragmento de borde de un plato de forma DRAG. 17 a con ruedecilla, de cerámica aretina lisa.

48. - Fragmento de base con arranque del pie de una copa aretina de la forma 11. Inv.: SPI-447.

49. - Base con arranque de las paredes de un vaso de cerámica aretina lisa, marca «in planta pedis» inscrita en circunferencia central: CN.ATE.PL. Del taller de Cn. Atei PLOCAMI (Oxé, 171, 1 c.h).

Diámetro de la base: $4,8 \mathrm{~cm}$. Inv.: SPI-428.

50.-Fragmento de base con parte de la pared de un plato de forma DRAG. 17 de cerámica aretina lisa. En el centro, restos de marca «in planta pedis»: EL. Posiblemen- 

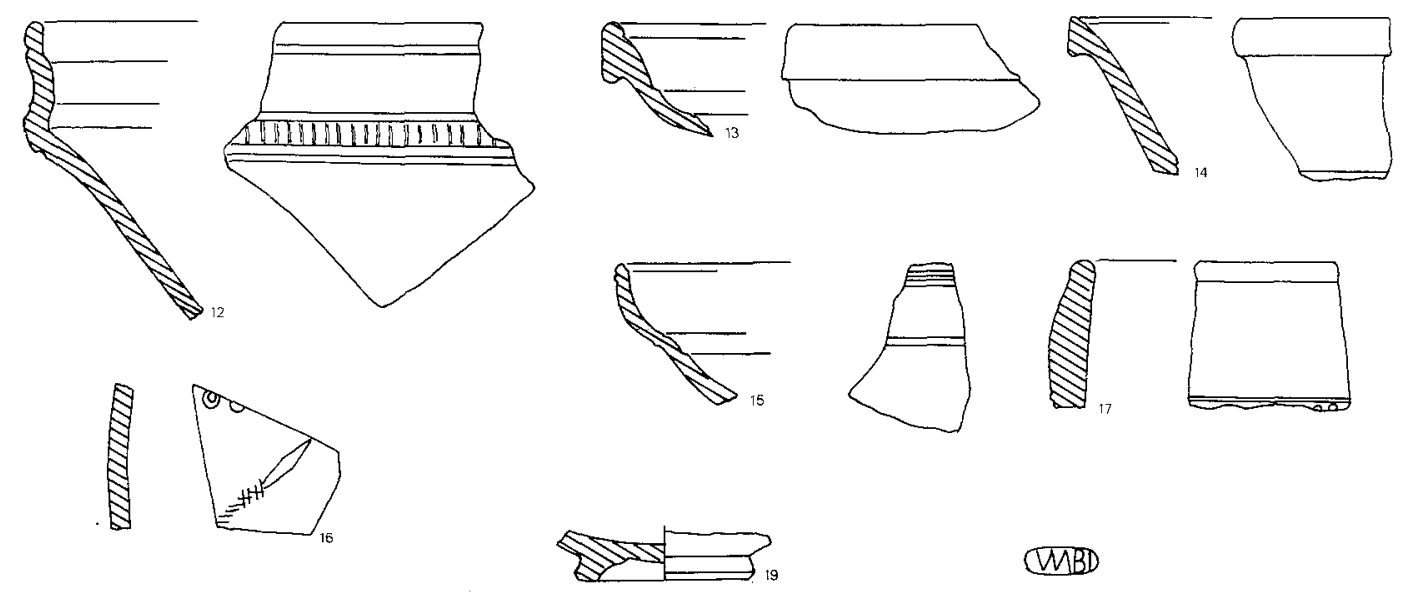

WBD
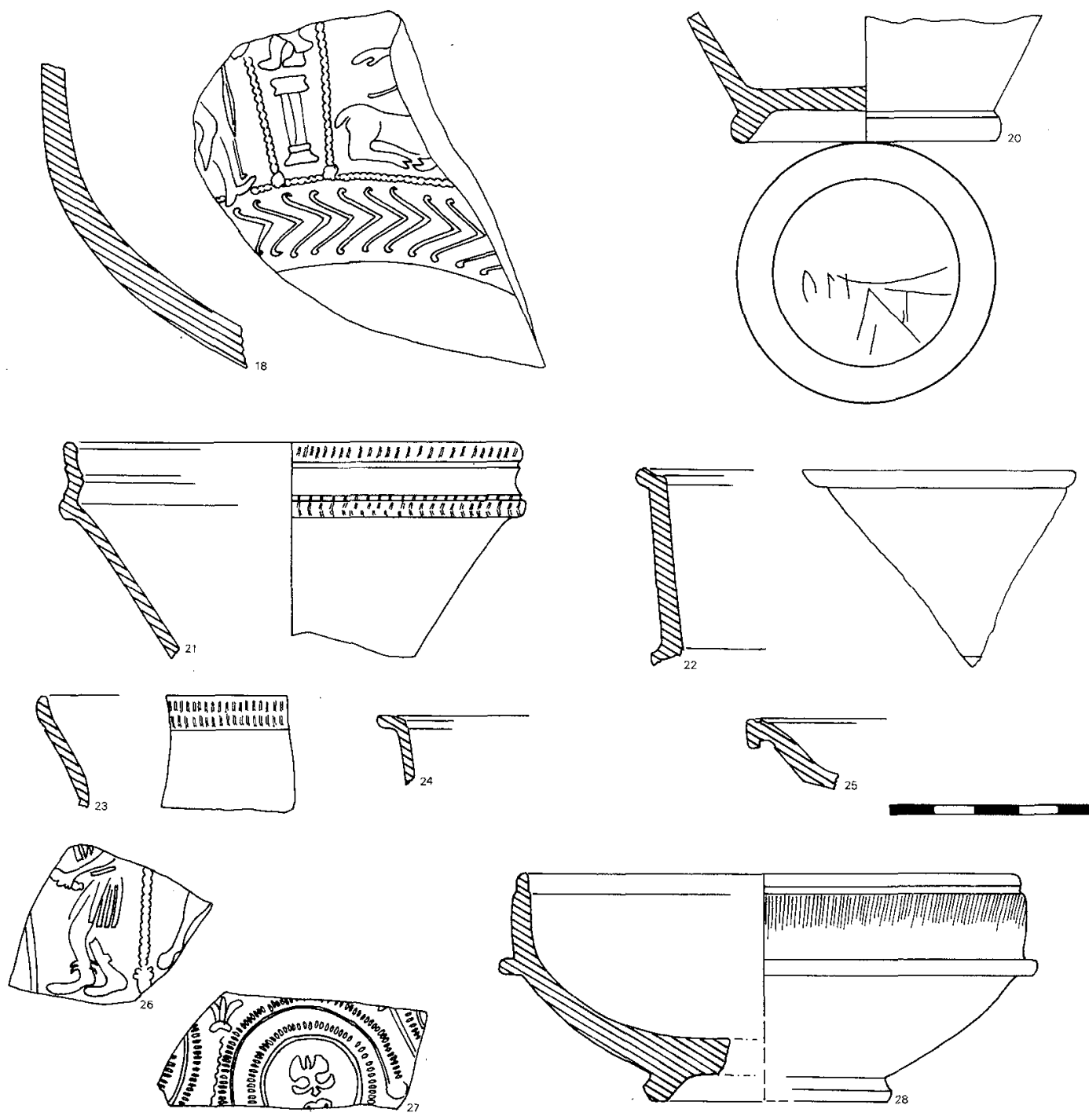

Fig. 6. Terra sigillata roja (aretina, sudgálica e hispánica). 

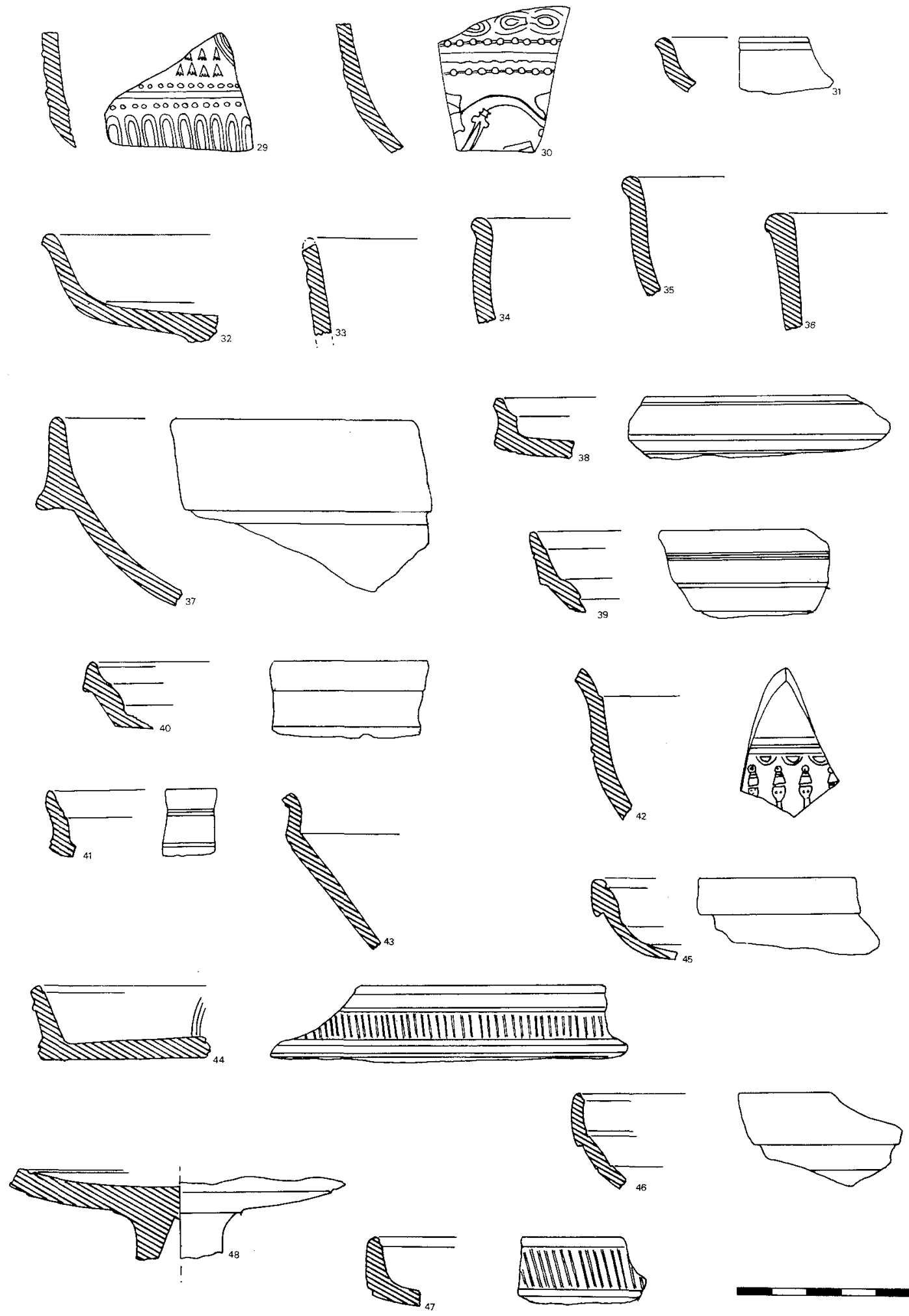

Fig. 7. Terra sigillata roja (aretina, sudgálica e hispánica). 

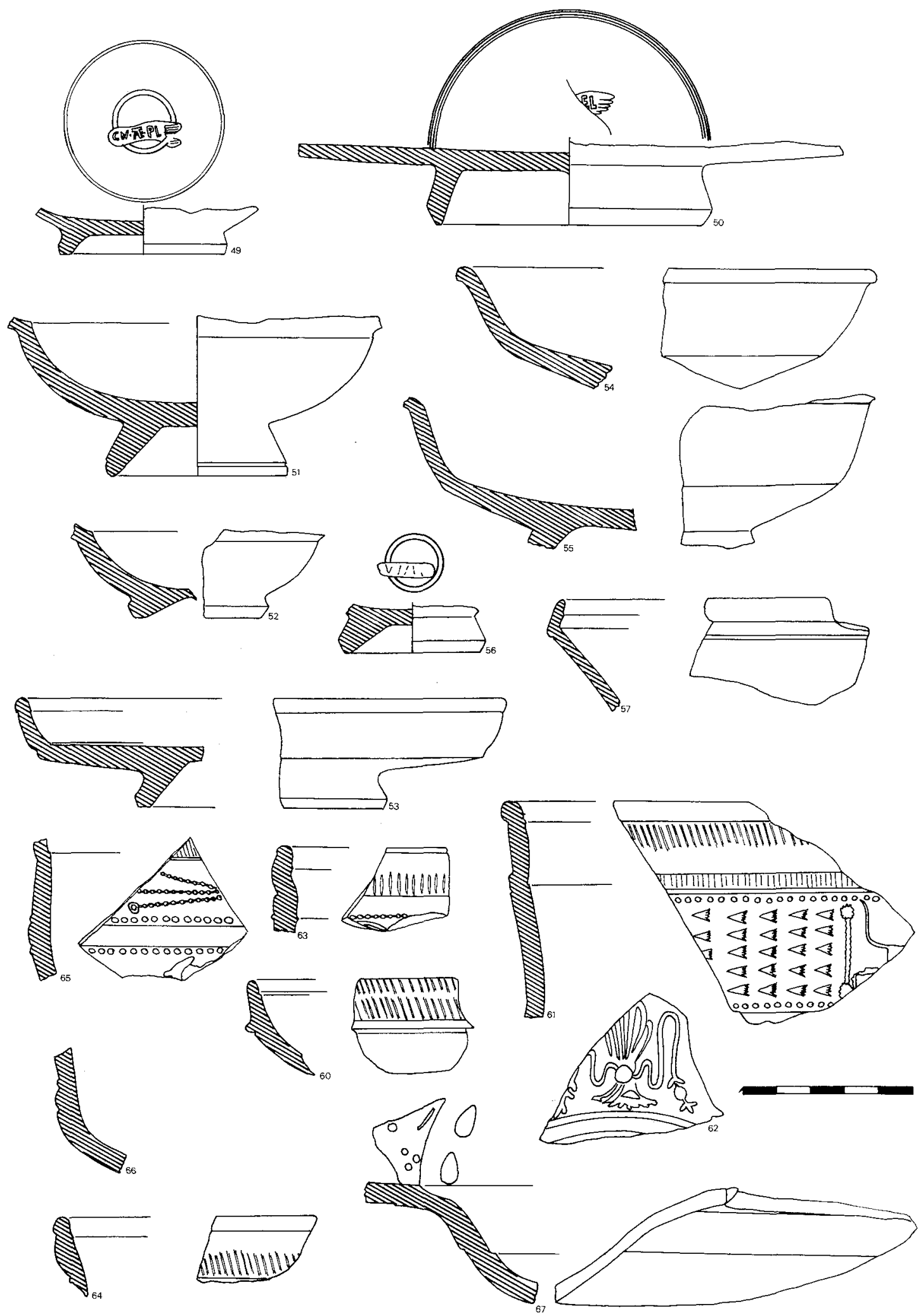

Fig. 8. Terra sigillata roja (aretina, sudgálica e hispánica). 
te, del taller de GELLIUS (Oxé, 736, 54.72 a.b).

Diámetro de la base: $7.7 \mathrm{~cm}$.

Inv.: SPI-427.

51. - Base y parte del primer cuerpo de una copa de forma DRAG. 27 de cerámica $s u d$ gálica lisa. Marca ilegible en cartela rectangular con los ángulos redondeados, inscrita en doble circunferencia.

Diámetro de la base: $5,2 \mathrm{~cm}$.

Inv.: SPI-439.

52.-Fragmento de una copa de forma DRAG. 27 de cerámica hispánica lisa. Inv.: SPI-446.

53.- Fragmento de plato de la forma DRAG. 18 (?) de cerámica sudgálica marmorata. Inv.: SPI-437.

54.--Fragmento de borde de un plato de la forma DRAG. 18 (?) de cerámica sudgálica lisa.

Inv.: SPI-436.

55.-Fragmento de borde, pared y arranque del pie de un plato de forma DRAG. 18 (?) de cerámica sudgálica lisa.

Inv.: SPI-434.

56. - Base de cerámica sudgálica lisa, con marca en cartela rectangular ilegible dentro de una circunferencia: VI...

Inv .: SPI-440.

57.-Fragmento de una copa RITT. 5 de cerámica sudgálica lisa. Inv.: SPI-449.

58.-Fragmento de una forma DRAG. 18 (?), de cerámica sudgálica lisa. Inv.: SPI-430.

59. - Fragmento de borde de un vaso de cerámica sudgálica o hispánica. Inv.: SPI-457.

60. - Fragmento de borde de una copa de forma DRAG. 24/25 de cerámica sudgálica lisa.

Inv.: SPI-450.

61.-Fragmento de borde y pared de una forma DRAG. 29 ó 30 de cerámica sudgálica decorada.

62.-Fragmento de la parte inferior de una forma DRAG. 29 de cerámica sudgálica decorada. Inv.: SPI-435.

63. -Fragmento de borde de una forma DRAG. 29 de cerámica sudgálica decorada. Inv.: SPI-438.

64.-Fragmento de borde de una forma DRAG. 29 de cerámica sudgálica decorada. Inv.: SPI-451.

65.--Fragmento de pared de una forma DRAG. 29 a de cerámica sudgálica decorada. Inv.: SPI-442.

66. - Fragmento de pared con carena de una forma DRAG. 29 de cerámica sudgálica decorada.

Inv .: SPI-432.

67.-Fragmento de pared con parte del borde decorado internamente, de una forma DRAG. 39 de cerámica hispánica lisa (Mezquiriz, lám. 17, A).

Inv.: SPI-443. 


\section{La Terra sigillata clara}

Este grupo cerámico, que arranca de fines del I d. C., es sabido que inaugura una nueva vajilla de lujo en el Mediterráneo central y occidental, mientras las áreas continentales van a seguir desarrollando la tradición de las sigillatas rojas. Dividida en diversas especies por el Prof. Lamboglia, Hayes integra los tipos A, C y D bajo el calificativo común de «African red slip ware», con una nueva nomenclatura de las formas. Seguiremos la doble denominación de estos autores, sin considerar las divisiones y nomenclatura de otros como Waagé y Salomonson, dado el cometido de este trabajo.

El lote que se presenta a continuación muestra notorias novedades, que creemos serán confirmadas por los últimos conjuntos procedentes de las actuales excavaciones en el Portus, como son la existencia de especies tales como la Clara B, lucente y la decorada con relieves aplicados, las cuales venían siendo consideradas por G. Martín como muy raras o inexistentes, según los casos, en el Levante y Sudeste peninsular.

La totalidad de los fragmentos proceden de las Balsas 1 y 2 , a excepción del núm. 524 (lucente), y el núm. 522 (Clara D) procedentes ambos de un mismo estrato: el nivel I de la zona A.

\section{Clara A}

De la forma Lamboglia 1-Hayes 8 poseemos cuatro fragmentos, dos de ellos con decoración a ruedecilla, mientas los otros son lisos.
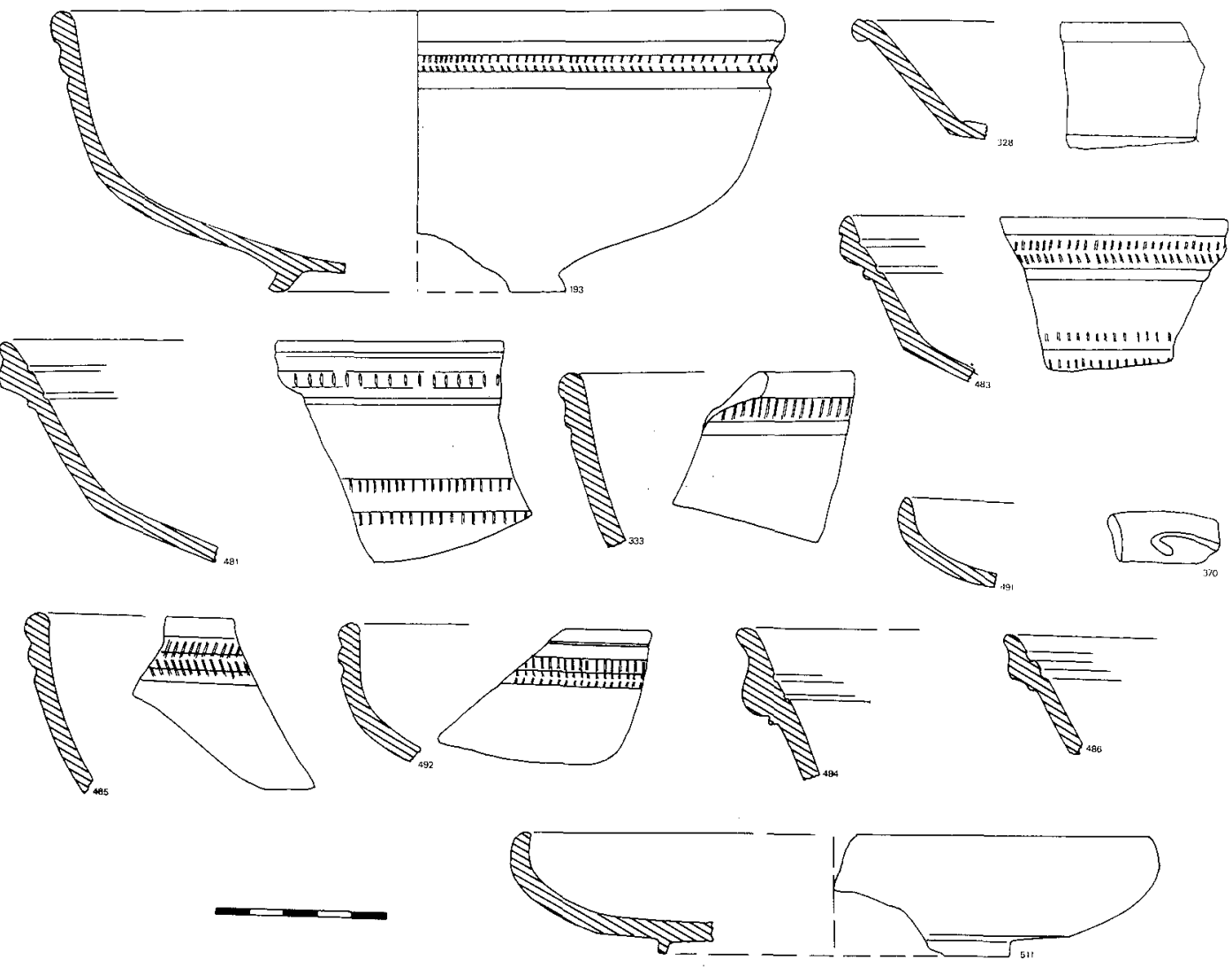

Fig. 9. Terra sigillata clara (A). Formas Hayes 8, 9, 3B, 5 y 27. 
Otros cuatro fragmentos representan a la forma Lamboglia 2A-Hayes 9, con datación idéntica a la de los vasos anteriores.

Hay un solo fragmento de la forma Lamboglia 4/36A-Hayes 3B con decoración de barbotina en el ala, mientras el núm. 328 corresponde a una forma Lamboglia 18Hayes 5 , de cronología flavia.

Finalmente, dos fragmentos de la forma Lamboglia 9-Hayes 27, que se data por el primero entre fines del II e inicios del III, basándose en la estratigrafía de Albintimilium (estratos IIIA y IIIB).

\section{Clara B}

Parece ser que el centro productor de esta especie cerámica, de pasta más clara y tierna, que se raya fácilmente con la uña y tinta la mano, se localiza en el valle medio del Ródano, en donde hubieron de existir varios talleres. De uno de ellos poseemos en el Portus de Illici dos medios vasos, que, aunque los hemos englobado dentro de la forma Lamboglia 1/3, en realidad representan la forma Drag. 17 B de la cerámica itálica ya recogida por la Clara A. La pasta tizna y el barniz anaranjado pálido, a pesar de un leve reflejo, no alcanza el grado iridiscente que personaliza a la cerámica lucente.

Esta especie se data entre 150 y 250 , fecha que otorga Lamboglia al estrato IIIB de Ventimiglia y al II de Ampurias.

\section{Lucente}

La cerámica «lucente» representa una vuelta al gusto por la superficie de brillo metálico que había sido propio de la cerámica romana republicana. Su radio de difusión -como es el caso de la Clara B- se limita al ángulo NO. del Mediterráneo, con escasísimos ejemplares más abajo de Cataluña, por lo que los hallazgos port-ilicitanos vienen a representar un importante jalón que permite ampliar el alcance comercial de tan peculiar tipo cerámico.
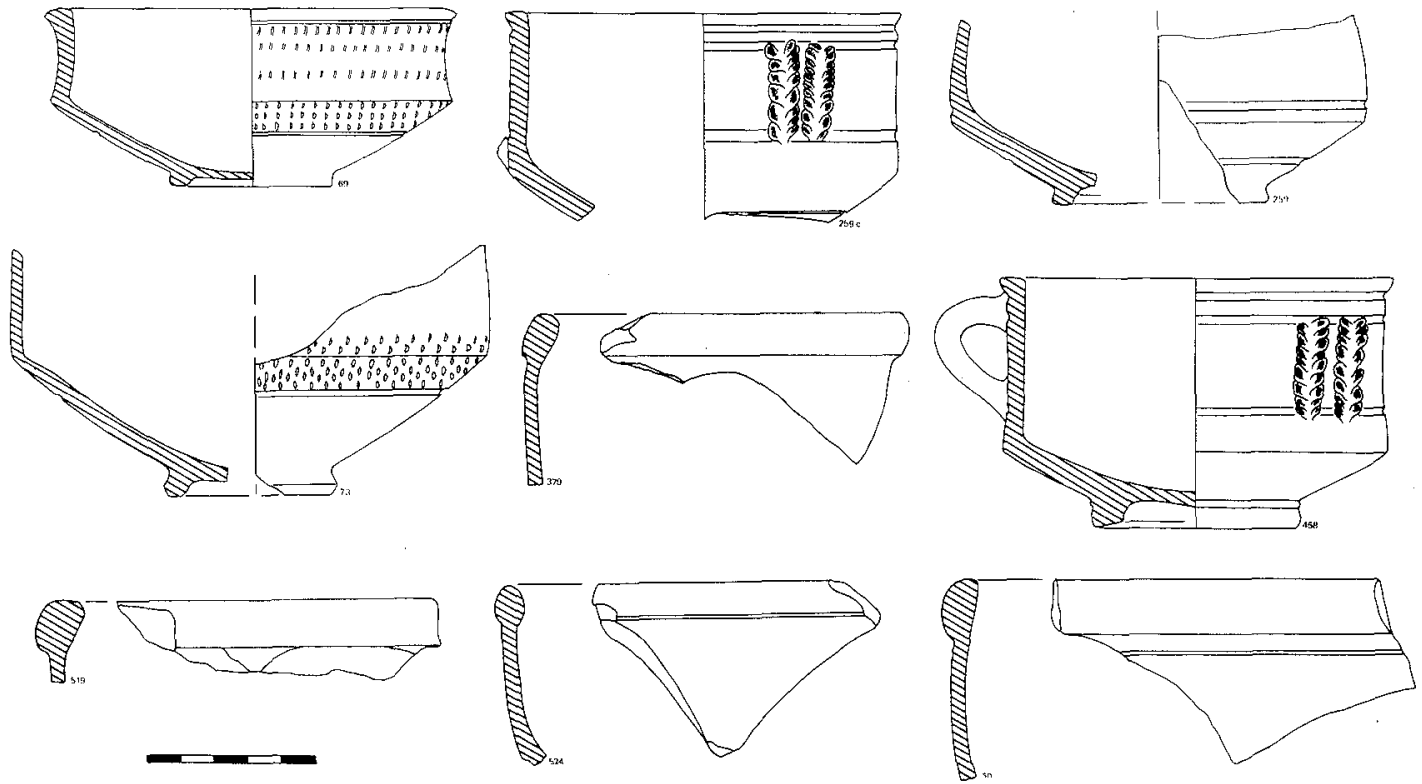

Fig. 10. Terra sigillata clara B y lucente. 

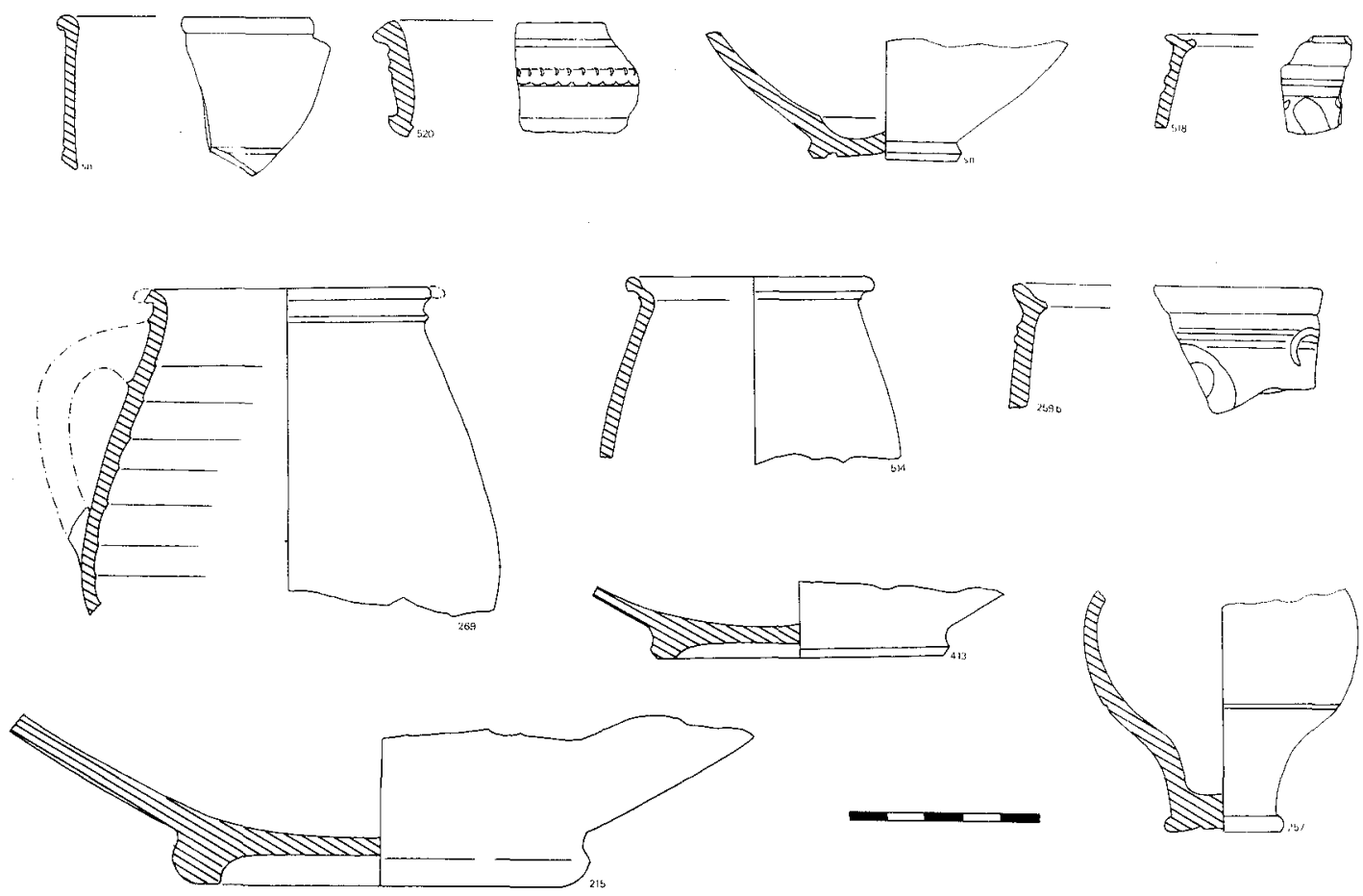

Fig. 11. Terra sigillata lucente.

Las verdaderas formas $1 / 3$ y $2 / 37$ de Lamboglia están bien representadas por varios fragmentos, con el típico borde almendrado. De nuevo, la forma Drag. $17 \mathrm{~B}$ la recoge el núm. 520, mientras dos tazas se incluyen como variantes de las formas $1 / 3$ y $2 / 37$ y nos ofrecen borde sencillo y una decoración estampada en forma de doble espiga.

La forma 14/26 de Lamboglia, característica según este autor del siglo IV, aparece también representada dentro de este grupo, con ejemplos de decoración a barbotina, junto con la parte inferior de un jarro de la forma 28 y otro fragmento de una copa de forma 45.

\section{Clara C}

Unicamente poseemos siete escasos fragmentos procedentes de las Balsas. De su más típica forma, Lamboglia 40-Hayes 50, hay cuatro fragmentos. Según el primer autor, se dataría en la segunda mitad del siglo III, mientras Hayes la sitúa un siglo más tarde.

La forma Lamboglia 41-Hayes 48A está presente con el fragmento núm. 475, y la forma Lamboglia 42 -Hayes 45 , con decoración de ruedecilla en la cara interna del borde, con dos fragmentos.

\section{Clara C y D con motivos aplicados}

En 1963, N. Lamboglia distinguía dentro de la Clara C una especie decorada con relieves aplicados que intentaba imitar la vajilla metálica decorada de esta guisa. Hoy parece evidente que su origen no es africano y derivaría, en cambio, de los vasos con apliques de medallones del Sur de Francia. 

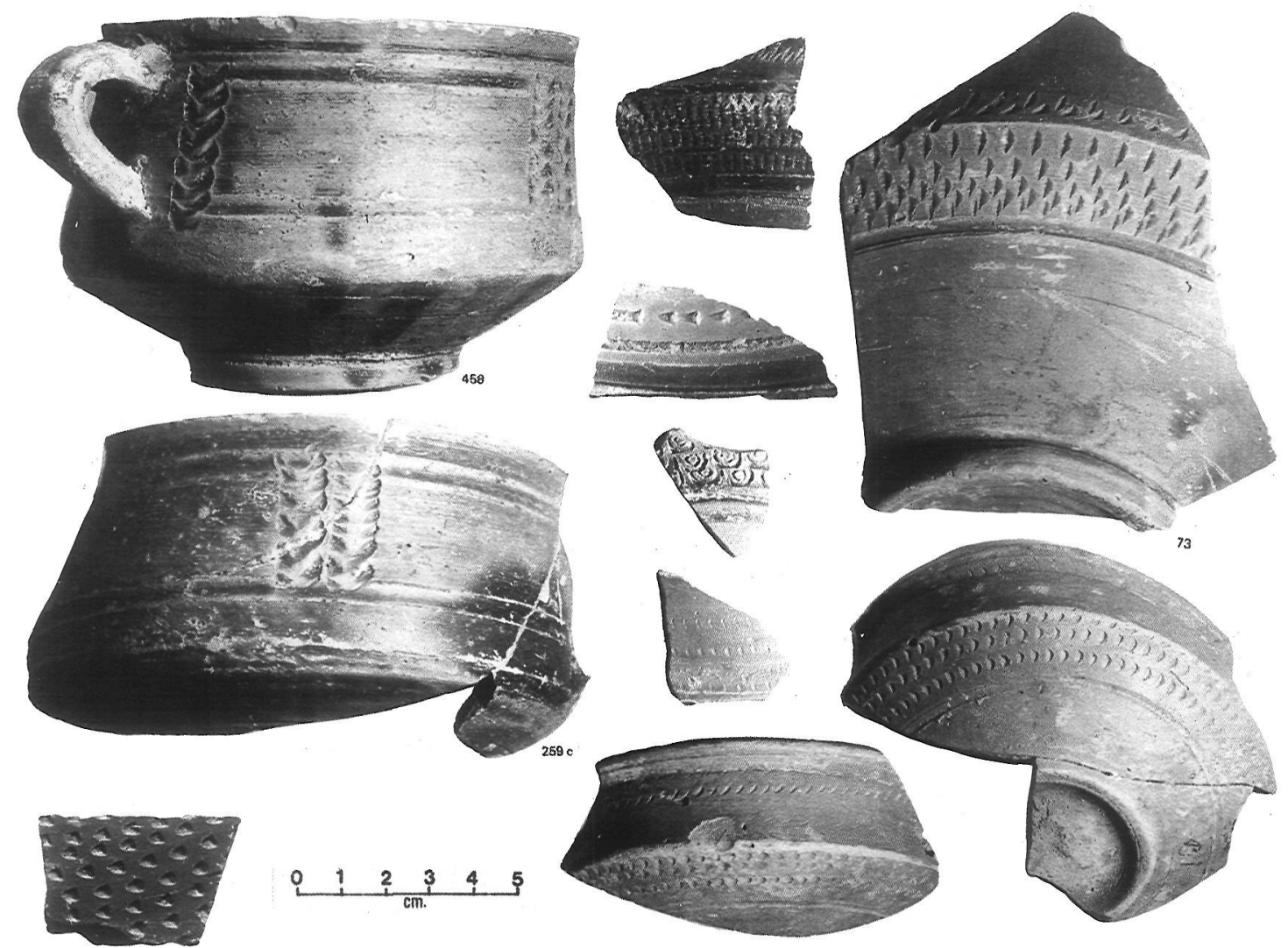

Lám. III. Cerámica clara B y lucente.

De los tres ejemplares port-ilicitanos, los pertenecientes a la forma Lamboglia 35Hayes $52 \mathrm{~B}$ entrarían en la Clara C, pero el representante de la fuente de borde poligonal (forma Lamboglia 42/48-Hayes 54) ofrece una textura de peor calidad, asimilable a la Clara D.

Los motivos representados son para la forma Hayes 52B los cuartos traseros de un felino (león o leopardo) y una palmeta, como en los ejemplares alicantinos que estudia Martín, y para la forma Hayes 97 la parte delantera de un lobo.

\section{Clara D}

Los ejemplares del Portus ofrecen todos las características pastas con el barniz más mate que los tipos precedentes, quedando sin cubrir el exterior del recipiente. Su calidad, no obstante, depende del lugar de hallazgo, ya que en las Balsas se acumuló un elevado grado de humedad que no sólo afectó al numerario de bronce.

Dentro del grupo de la sigillata anaranjada constituye la especie más numerosa y muchos fondos de los vasos, sobre todo grandes fuentes, posiblemente estén recogidos en el corpus de decoraciones estampadas. Por ello, su cronología irá también ligada a la que arroje la propia decoración estampada, es decir, entre los siglos IV y V.

La forma Lamboglia 9-Hayes 63 cuenta con cinco fragmentos de otras tantas fuentes, mientras seis ejemplares representan a la forma Lamboglia 24/25-Hayes, 91, en parte con decoración de ruedecilla en el interior del fondo.

Catorce fragmentos pertenecen a la forma Lamboglia 42-Hayes 67, y la forma Lamboglia 51-Hayes 59A y B está documentada con 19 ejemplares. De la forma Hayes 60 sólo existe un ejemplar. 

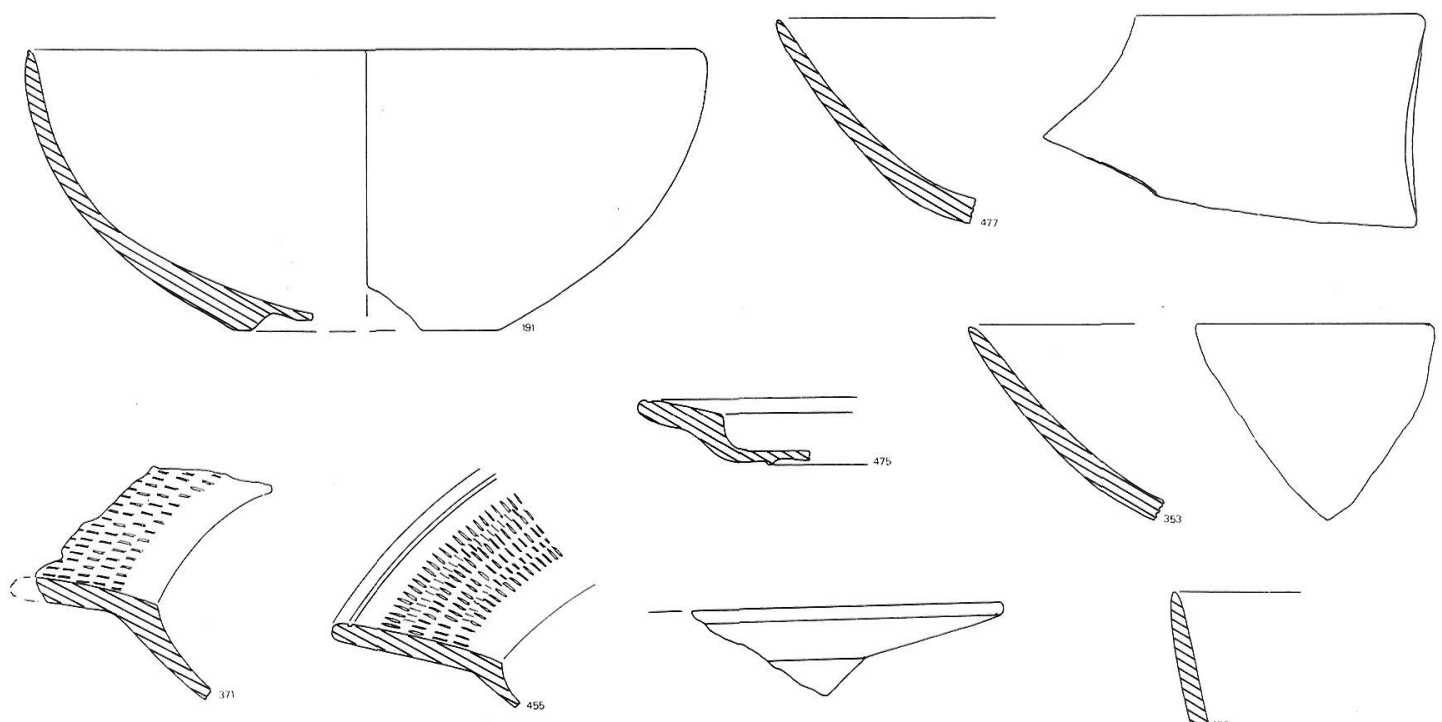

Fig. 12. Terra sigillata clara (C). Formas Hayes 50, 45 y $48 \mathrm{~A}$.

La forma Lamboglia 52-Hayes 58 es la más abundante, con 25 fragmentos, seguida de la forma Lamboglia 54-Hayes 61 A, con 20 fragmentos de borde triangular.

Otras formas se hallan poco representadas, como Lamboglia 55-Hayes $61 \mathrm{~B}$, con dos escasos ejemplos, y la forma Lamboglia 57 -Hayes 70, con uno solo. Por otro lado, es de destacar la ausencia de la forma Lamboglia 1-Hayes 99, más tardía, acompañada a menudo de temas cristianos estampados en su fondo.
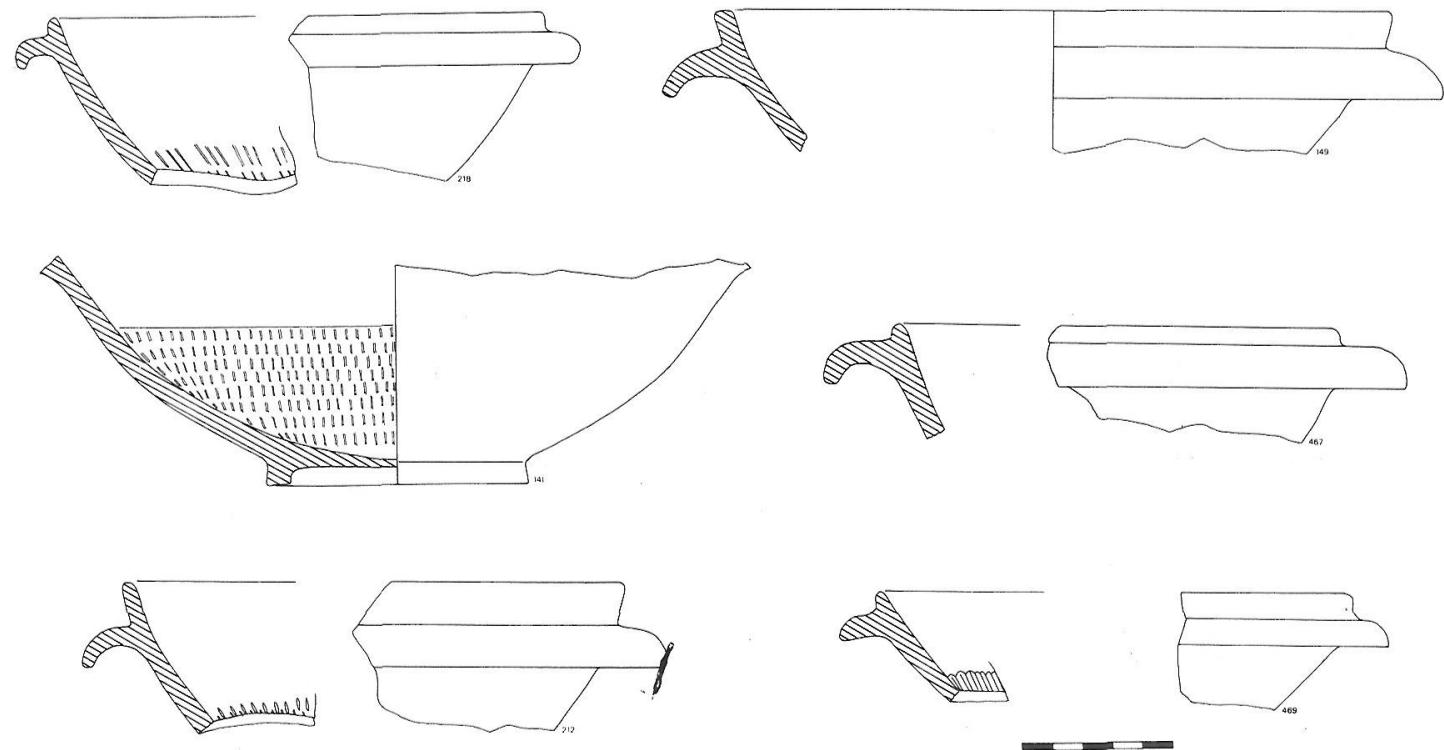

Fig. 13. Terra sigillata clara (D). Forma Hayes 91. 

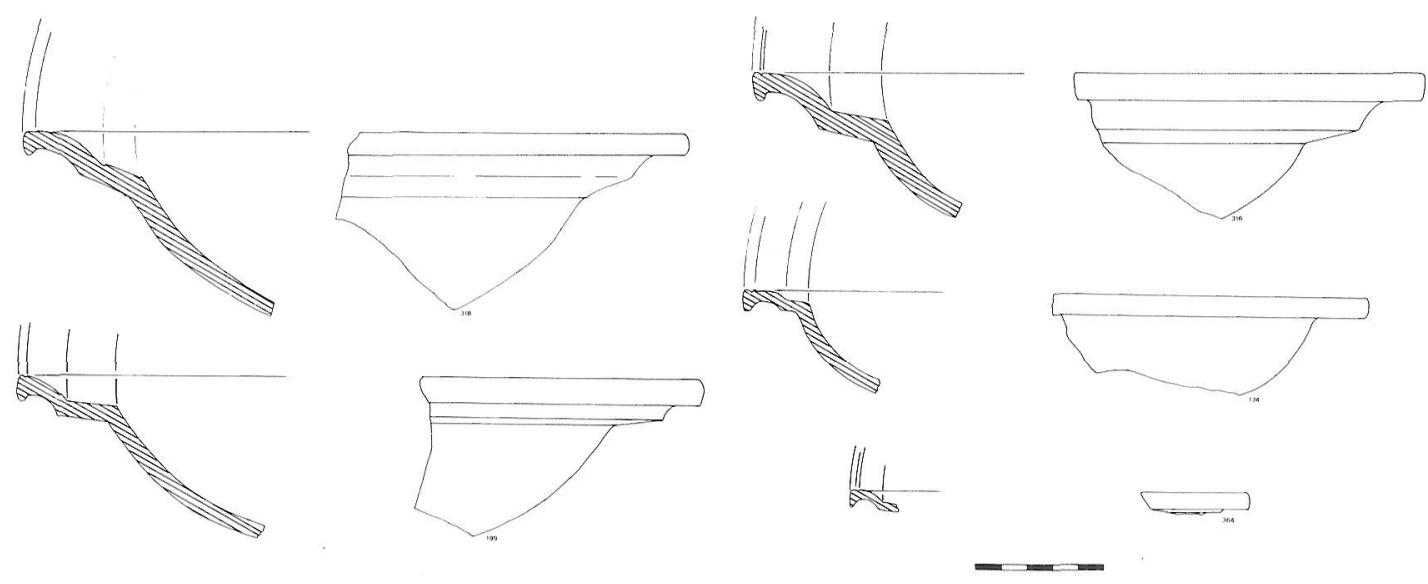

Fig. 14. Terra sigillata clara (D). Forma Hayes 67.

\section{Clara D estampada}

La totalidad de los fragmentos recuperados presenta una clara unidad decorativa, idéntica a la producción marroquí, con motivos geométricos bien conocidos: círculos concéntricos, palmas, rosetas de diversos tipos, crecientes y una forma geométrica con líneas internas entrecruzadas formando damero, que entrarían en los estilos A(i), A(ii), A(iii), B y C de Hayes, con una datación entre 340 y 440.

La identidad técnica con los ejemplares lisos es evidente y con total seguridad los fondos que aquí se recogen pertenecen a las formas 42, 51, 52 y 54 de Lamboglia arriba mencionados. Su descripción es la siguiente:

463.-Dos fragmentos del fondo de una fuente, decorado con palmetas radiales dentro de doble circunferencia.

290. - Fragmento de fondo con guirnalda compuesta a base de alternancia de crecientes rayados, enmarcando un conjunto de palmas radiales en torno de triple circunferencia central, todo ello enmarcado por doble circunferencia.
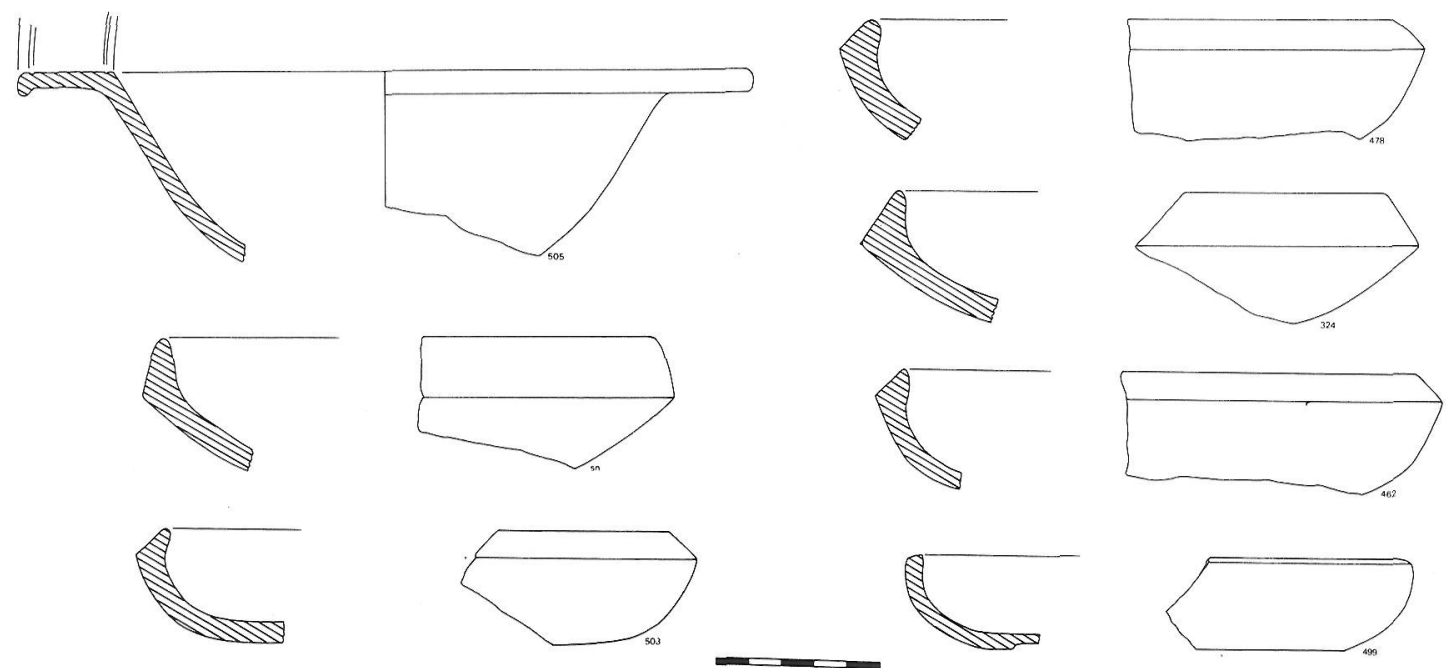

Fig. 15. Terra sigillata clara (D). Formas Hayes 70 y $61 \mathrm{~A}$. 

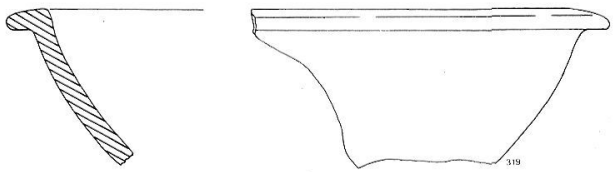

$\sin$
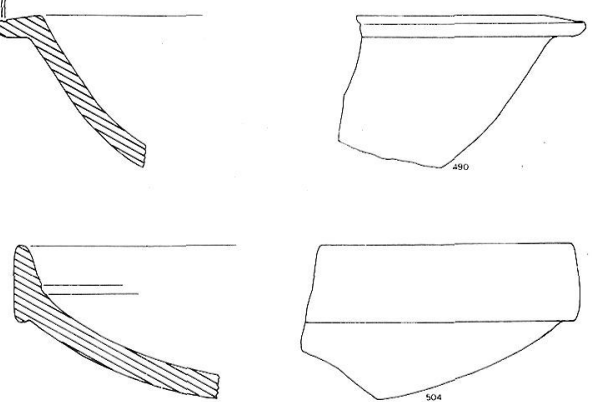
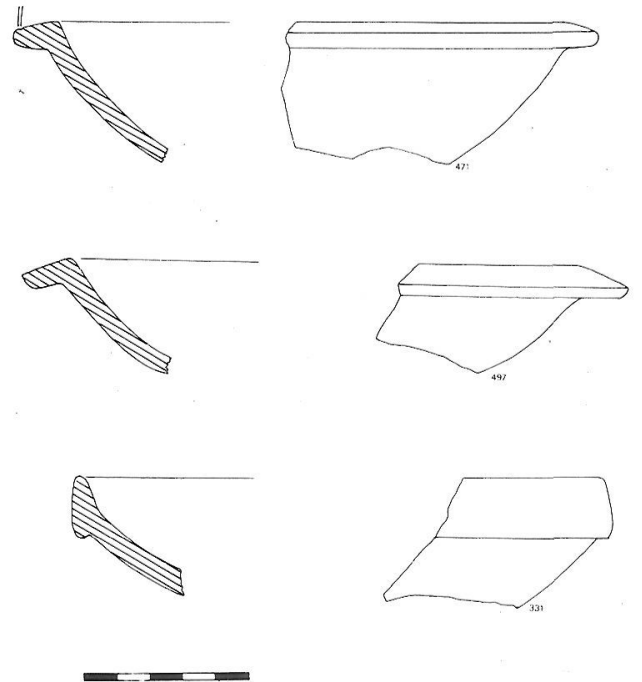

Fig. 16. Terra sigillata clara (D). Formas Hayes 61B y 58.

222. - Cinco fragmentos que conforman el fondo de una fuente decorado con grupo central de cinco palmetas con rosetas, enmarcado por cuádruple circunferencia, con orla de rosetas entre la segunda y la tercera.

242.-Tres fragmentos pertenecientes al fondo de una fuente, con decoración de un grupo central de palmetas radiales alrededor de doble circunferencia concéntrica y rodeado de pequeños circulillos concéntricos. Tras doble circunferencia, una cenefa de palmetas dispuestas oblicuamente da paso al último doble surco que enmarca la decoración.

305.-Tres fragmentos unidos que conforman parte del fondo, con decoración de orla de círculos concéntricos y rosetas alternantes, entre surcos.

291.-Dos fragmentos unidos de parte del fondo de una fuente decorada: dentro de triple surco, grupo de circulillos concéntricos y restos de palmeta.
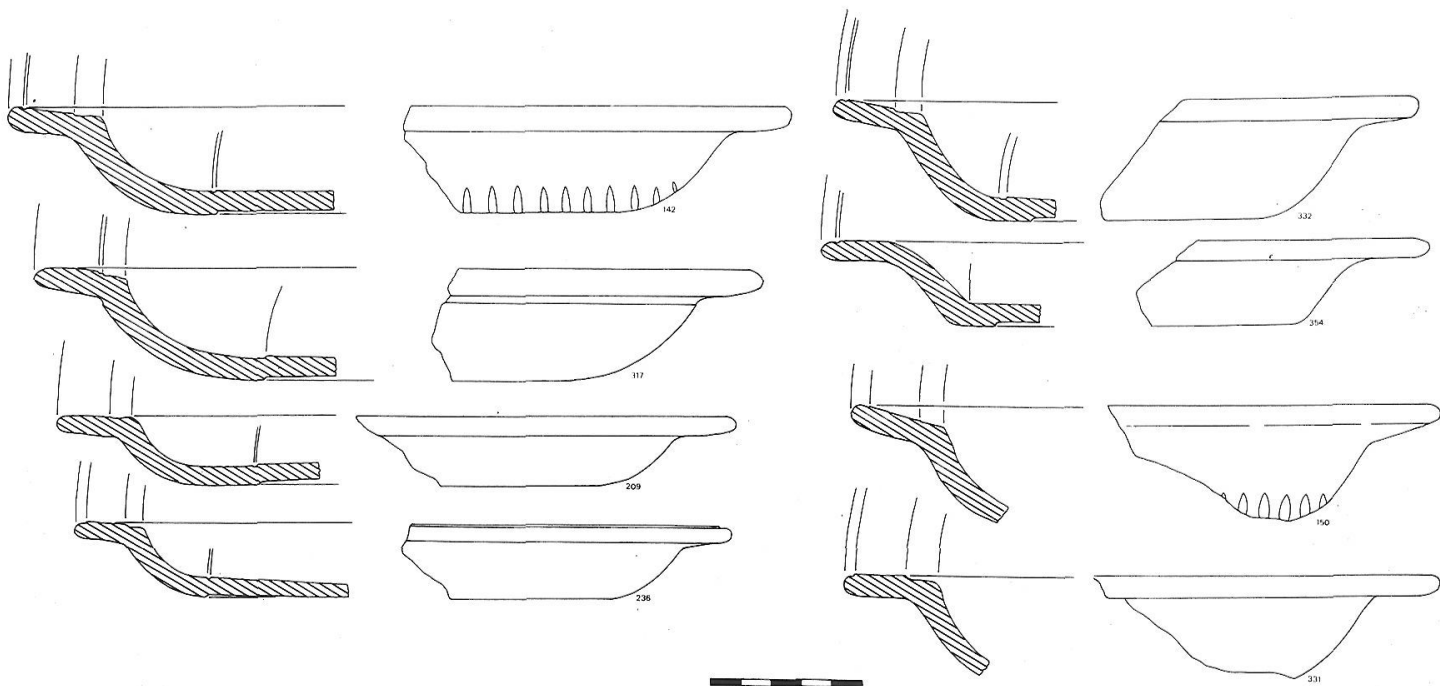

Fig. 17. Terra sigillata clara (D). Formas Hayes 59 y 60. 


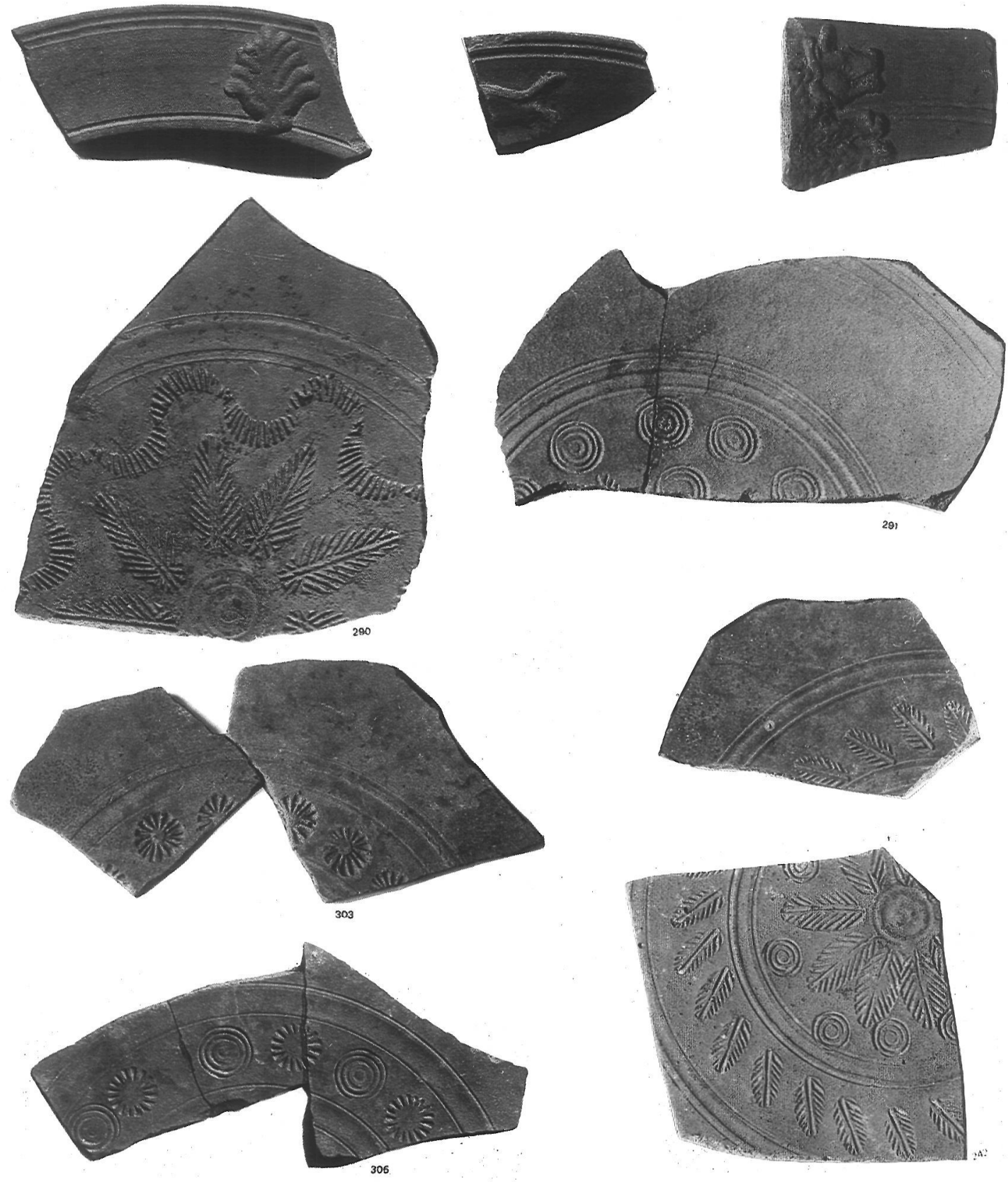

Lám. IV. Sigillatas clara C y D con relieves aplicados y con decoración estampada de tipo geométrico.

303. - Dos fragmentos del fondo de una fuente con orla de rosetas entre surcos concéntricos.

58.-Fragmento del fondo decorado de una fuente: orla de círculos concéntricos con pequeñas rosetas, entre dos surcos.

241.-Fragmento de fondo, con orla de rosetas dentro de doble circunferencia.

289. - Fragmento de fondo decorado: restos de grandes rosetas o crecientes en el interior de doble surco concéntrico. 


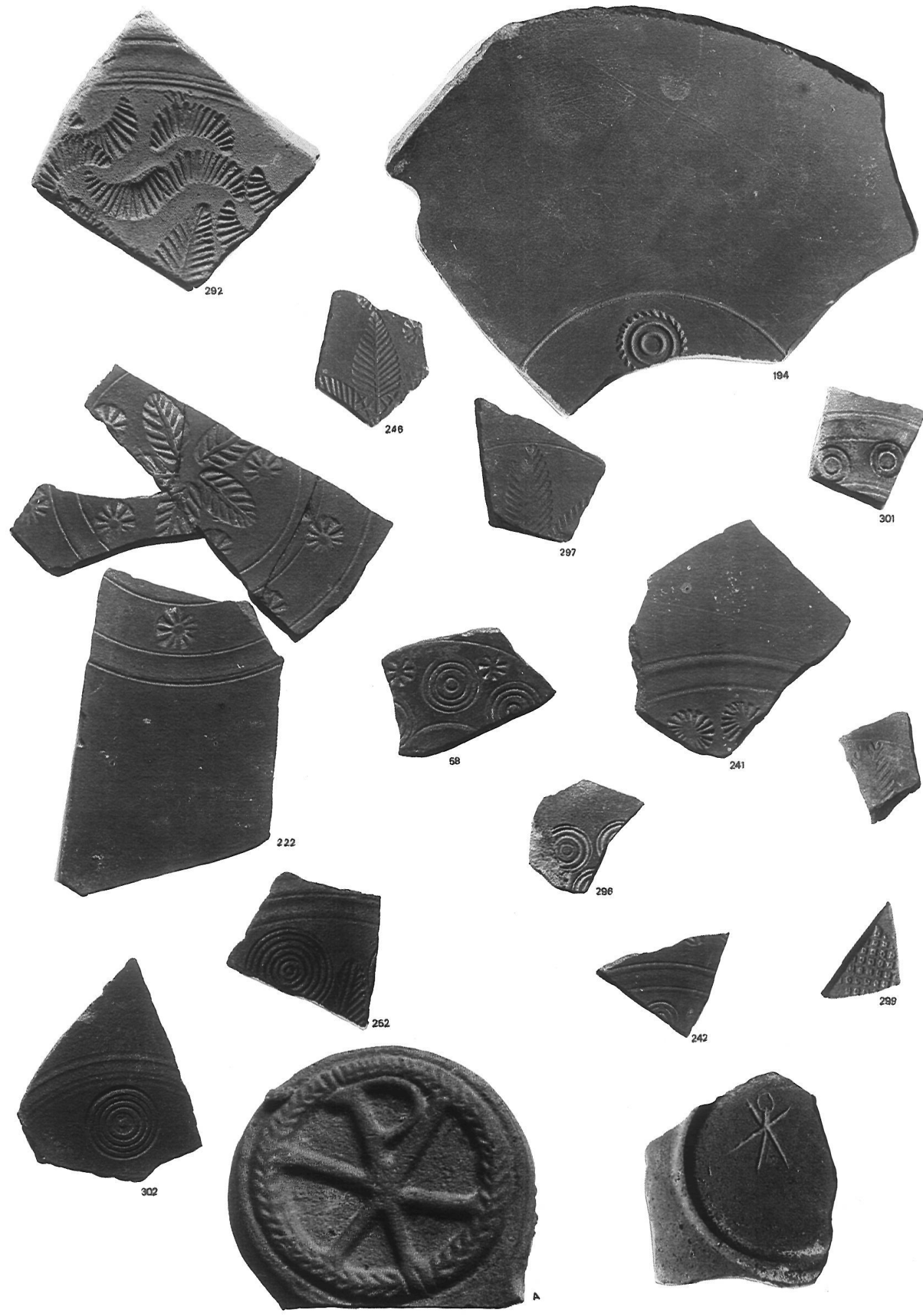

Lám. V. Sigillata anaranjada con decoración estampada. Disco reflector de lucerna paleocristiana y grafito con el crismón. 
194. - Fragmento de fondo decorado y pared, con círculo concéntrico con dentado espiral, dentro de circunferencia.

295. - Fragmento de fondo decorado: orla de circulillos concéntricos y rosetas alternantes, seguidos de doble surco que enmarcan un grupo central de palmetas (restos) y circulillos nuevamente concéntricos.

300.-Fragmento de fondo decorado: dentro de doble surco restos de palmetas radiales y entre ellas crecientes.

302.-Fragmento de fondo decorado: enmarcado con circunferencias un gran círculo concéntrico.

292. - Fragmento de fondo, con restos de un grupo central de palmetas radiales rodeado de crecientes enlazadas formando ondas.

246. - Fragmento de fondo con decoración de grupo central de palmetas radiales rodeadas de pequeñas rosetas.

297. - Fragmento de fondo decorado: restos de grupo central de palmetas radiales dentro de doble circunferencia.

298.-Fragmento con restos de una palmeta, en el interior de doble circunferencia.

252.-Fragmento de fondo decorado: en el interior de doble surco, restos de grupo de palmetas y grandes círculos concéntricos.

296. - Fragmento de fondo de una fuente con decoración de restos de grupo central de círculos concéntricos.

361.-Fragmento de fondo decorado: entre surcos, orla de circulillos concéntricos.

299. - Fragmento de fondo decorado: motivo estampado con interior cuadriculado.

\section{Sigillata gris}

Un solo ejemplo ha sido hallado en las excavaciones de 1976: un fragmento de borde de una escudilla o fuente de la forma 4 de Rigoir. Posiblemente el fondo se encontraría estampado.

Con una cronología que la mayoría de los autores sitúan en los siglos V y VI, es interesante recordar la hipótesis de Lamboglia según la cual este tipo de cerámica que vuelve al fuego reductor lejos de suceder a la Clara D lo haga de la Clara B tardía, con barniz más oscuro del tipo de la lucente, desprendiéndose de ello su contemporaneidad con la Clara D (10). La persistencia de la misma decoración que en la estampada roja, habla también en este sentido.

\section{Disco reflector de lucerna paleocristiana}

Aprovechamos este trabajo para dar a conocer un fragmento decorado de una lámpara cristiana. Se trata de un disco reflector decorado con orla de laurel que enmarca el Crismón. El parecido con el ejemplar de Tipasa es evidente (BARADEZ, 1954), viniendo a ser uno de los escasos ejemplos de iconografía cristiana proporcionados por el Portus Illicitanus. En fotogafía aparte recogemos otro fragmento de base de una pátera de sigillata clara D, en cuyo fondo externo se presenta grafitado un crismón.

\section{BIBLIOGRAFIA}

AL-HYMYARI, Kitab ar-rawd al-Mi'tar. Traducción del francés de Pilar Maestro. Anubar. Valencia, 1963.

BARADEZ, J., 1954, «Tipasa: disque réflecteur de lampe chrétienne».

BELTRAN LLORIS, M., 1978, Cerámica romana. Tipología y clasificación. Zaragoza. 
BALIL, A., 1983, «Terra sigillata africana de Els Antigons (Alicante)». IDEA, 40, 7-24. Alicante.

CABALLERO ZOREDA, L., 1966, «Estado actual del estudio de la cerámica sigillata clara en el Mediterráneo occidental». Ampurias, XXVIII. Barcelona.

- 1970, «Nuevos datos sobre cerámica sigillata hispánica, sigillata clara de tipo B y sigillata brillante», TP, 27. Madrid.

- 1971, «Cerámica sigillata clara decorada de los tipos A, A/C y C». TP, 28. Madrid.

- 1974, «Cerámica sigillata clara de tipo D estampada de las provincias de Murcia y Almería», Miscelánea Arqueológica, I. Barcelona.

ESCOLANO, G., 1879, Décadas de la Historia de la insigne y coronada ciudad y reino de Valencia, tomo II. Ed. Terraza, Aliena y Compañía. Valencia.

FONTES HISPANIAE ANTIQUAE, vols. VIII y IX. Universidad de Barcelona.

GONZALEZ PRATS, A., 1977, «Materiales para un conocimiento del Portus Illicitanus, I: las lucernas». ITEM, 4. Alicante.

HAYES, J. W., 1972, Late roman pottery.

IBARRA, A., 1879, Illici, su situación y antigüedades. Alicante.

IBARRA, P., 1962, Elche, materiales para su historia. Cuenca.

JODIN, A.-POSCHIN, M., 1960, «La céramique estampée du Maroc romain». B. A. M., IV. Rabat.

- 1967, «Nouvelles observations sur la céramique estampée du Maroc romain», $B . A . M$., ViI. Rabat.

LAMBOGLIA, N., 1958, «Nuove osservazioni sulla terra sigillata chiara (Tipi A e B)». R. S. L., XXIV. Bordighera.

- $\quad$ 1963, «Nuove osservazioni sulla terra sigillata chiara (II)». R. S. L., XXIX. Bordighera.

LLOBREGAT, E. A., 1973, Teodomiro de Oriola. Su vida y su obra. Alicante.

- 1983, «Relectura del Ravennate: dos calzadas, una mansión inexistente y otros datod de la geografía antigua del País Valenciano». Lucentum, II, 225-242. Alicante.

MARTIN, G., 1968, «Comercio y producción de cerámicas finas en época imperial». PLAV, 5. Valencia.

- 1975, «Un vaso de sigillata clara en el Museo de Alicante». IDEA, 25, 109-126. Alicante.

- $\quad 1979$, «Problemas de metodología, cronología y difusión de algunos tipos de sigillata clara y su localización en la provincia de Alicante». IDEA, 28, 7-76. Alicante.

MEZQUIRIZ, M. A., 1961, Terra sigillata hispánica. Vols. I-II. Valencia.

OXE, A.-OSWALD, F., 1914, Die ältesten Terra Sigillata-Fabriken in Montans am Tarn. Arch. Anzeiger, XXIX. Berlín.

OXE, A.-COMFORT, J., 1968, Corpus Vasorum Arretinorum. A Catalogue of the Signatures, Shapes and Chronology of Italian sigillata. Bonn.

OSWALD, F.-PRYCE, T. D., 1966, An Introduction to the study of Terra sigillata. Treated from a Chronological stadpoint. London.

PALOL, P. de, 1948, «La cerámica estampada romano-cristiana». IV, CASE, 450-469.

RAMOS FOLQUES, A., 1974, Santa Pola y su historia. Elche.

RAMOS FERNANDEZ, R., 1975, La ciudad romana de Illici. Alicante.

SANCHEZ FERNANDEZ, M. ${ }^{a}$ J., 1983, «Cerámica común romana del Portus Illicitanus». Lucentum, II, 285-317. Alicante.

Artículos aparecidos en diversos diarios durante la realización de la campaña de urgencia:

- «Posible localización del Portus Illicitanus, en Santa Pola» (INFORMACION, 15-XII-76).

- «Adquieren más importancia los descubrimientos arqueológicos» (INFORMACION, 17-XII-76).

- «Se cambia historia por un bloque de apartamentos» (LA VERDAD, 19-XII-76).

- «Se intensifican las excavaciones arqueológicas» (INFORMACION, 24-XII-76).

- «Descubrimiento de una ciudad romana en Santa Pola (Alicante)» (EL PAIS, 24-XII-76).

- «Que no se consume. la destrucción» (INFORMACION, 28-XII-76).

- «Consternación por el antiguo Portus Illicitanus» (LA VERDAD, 28-XII-76).

- «En apoyo de la conservación del Portus Illicitanus» (LA VERDAD, 31-XII-76).

- «Se ha confirmado la ubicación del famoso yacimiento arqueológico del Portus Illicitanus» (ABC, 2-I-77).

- «El Instituto de Estudios Alicantinos, contra la destrucción del patrimonio arqueológico» (INFORMACION, 4-I-77).

- «La destrucción del Portus Illicitanus» (INFORMACION, 5-I-77).

- «Santa Pola: nadie quiere destruir el Patrimonio arqueológico local» (INFORMACION, 6-I-77).

- «Se construirá sobre el Portus Illicitanus» (LA VERDAD, 14-I-77).

- «Comenzó la destrucción del Portus Illicitanus» (INFORMACION, 14-I-77).

- «Repulsa por la destrucción del Portus Illicitanus» (Cartas al Director, en INFORMACION, 18-I-77).

- «Suspendidas las obras en el Portus Illicitanus» (LA VERDAD, 18-I-77).

- «El Patrimonio Artístico suspende las obras realizadas sobre el Portus Illicitanus» (ABC, 18-I-77).

- «El Portus Illicitanus depende de Madrid» (INFORMACION, 15-I-77). 